\title{
Attitude Decoupling Control of Semi-floating Space Robots Using Time-delay Estimation and Super-twisting Control
}

\author{
Xin Zhang, Member, IEEE, Jinguo Liu, Senior Member, IEEE, Yuchuang Tong, Student Member, IEEE, Yuwang \\ Liu, Member, IEEE, Zhaojie Ju, Senior Member, IEEE
}

\begin{abstract}
Dynamic coupling is an inherent behavior of the space robot system, which seriously affects the motion accuracy of the system. This paper focuses on how to guarantee the base attitude stability of semi-floating space robots, from a control perspective, namely, the base attitude decoupling control. The quaternion representation of the system dynamics is derived to avoid the representation singularity of the Euler angles. In consideration of the complicated system dynamics and the limited computing ability of the on-board computer, an efficient decoupling controller is designed based on the time-delay estimation (TDE) and the super-twisting control (STC). Herein, for the proposed TDE-based STC (TDE-STC) scheme, TDE is used to decouple and linearize the nonlinear dynamics, and STC is a second-order sliding mode control (SMC) algorithm which can compensate for the TDE error and drive the state variables to converge to the equilibrium point robustly in finite time. The model-free decoupling principle of TDE is clearly illustrated by comparison with the classical computed-torque control (CTC). The global asymptotical stability analysis of the closed-loop system is proven using the Lyapunov theory and the linear matrix inequality (LMI) theorem. Finally, several comparative simulation studies on a three-dimensional (3-D) space robot system with consideration of joint friction, disturbances, and model uncertainties are conducted to verify the effectiveness of the proposed TDE-STC scheme. The corresponding results show that the TDE-STC scheme can achieve a high-accuracy attitude decoupling performance with both the model-free merits and the chatter-free merits.
\end{abstract}

Index Terms-Semi-floating space robots, Base attitude decoupling control, Quaternion representation, Time-delay estimation, Super-twisting control.

\section{INTRODUCTION}

$\mathbf{F}$ Or large space structures, such as space trusses and solar arrays, they need to be monitored periodically for possible damage [1]. Space robots are satellite-manipulator structures [2] possessing the mobility of satellites and the dexterous operability of robotic arms [3], which are suitable for executing the on-orbit capture [4], inspection [5], damage detection, and health monitoring tasks [1]. Unlike basefixed robots, non-fixed satellite bases have a special behavior, namely, the dynamic coupling, which usually results in severe disturbances on the base and the operational accuracy of the end-effector. For on-orbit robotic detection missions, the space robot gradually approaches the large structure, and then the manipulator carries various measuring instruments [6]-[8] to detect and locate the damage source. During this period, the base position is not controlled, but the pointing accuracy of the end-effector and the attitude stability of the base should be guaranteed simultaneously during the operation [9], [10]. Herein, we term this partial base control mode [11] as semifloating mode in which the base attitude and the manipulator are under control but the base position is not controlled. As the dynamic coupling will bring about adverse effects, developing effective methods of counteracting or compensating for the dynamic coupling has attracted lots of interest in the field of space robotics.

Over the past decades, researchers have investigated some pioneering studies on the dynamic coupling theory. For example, $\mathrm{Xu}$ and Shum [12] analyzed the coupling effect of the space robot system with a single arm and proposed the concept of the coupling factor to describe the motion relationship between the manipulator and the base. Zhou et al. [13] analyzed the dynamic coupling effect of the multi-arm space robot, and proposed the dynamic coupling ellipsoids for measuring the degree of coupling. $\mathrm{Xu}$ et al. [14] divided the dynamic coupling into two sub-problems, namely, the position coupling and the attitude coupling. The position coupling equation is a holonomic constraint that can be integrated to obtain the motion equation in the position level, but the attitude coupling equation is a nonholonomic constraint that is not integrable. Furthermore, some kinematic planning and control approaches, such as the reaction null-space (RNS) methods [15]-[17] and the dynamic balance method [18], have been proposed to address the problem of attitude coupling, and these approaches map the base attitude motion into the null-space of the Jacobian matrix of the manipulator. Thus, the kinematical redundant degrees-of-freedom (DoFs) of the manipulator is a prerequisite for the reactionless control strategies.

In practice, the momentum exchange devices consume the renewable electrical power; thus, compensating for the base attitude disturbance is feasible for space robots. In comparison to the RNS planning and control strategies, the active decoupling control strategy gets rid of the redundancy constraint of the manipulator, which is a more direct approach for stabilizing the base attitude. Longman et al. [19] adopted the dynamic analysis method to obtain the coupled force and torque that can be used as a feedforward compensation control signal into the base control system. In this method, the NewtonEuler recursive method is used to calculate the inner force and torque between the manipulator and the base; however, the Lagrange's modelling method is not applicable because the Lagrange's method is designed to eliminate the inner constraint force and torque. Furthermore, Antonell et al. [20] 
used the same principle to realize the coordinated control of the satellite-manipulator system. Jayakody et al. [21] proposed a diagonalization and virtual control technique to decouple the space robot system and used SMC to realize the coordinated motion control of the base attitude and the manipulator. Similarly, Shi et al. [22] used the diagonalization technique to decouple a dual-arm space robot system, and developed three controllers based on the CTC framework to coordinate the base attitude and the robotic arm motion, respectively. Zong and Emami [23], [24] proposed a concurrent control strategy that makes the space robot rendezvous with a target and the manipulator and the base attitude track desired trajectories simultaneously. Zhu et al. [25] proposed an adaptive sliding mode disturbance observer to realize the attitude stability control during the space robot capturing targets. Muralidharan and Emami [5] proposed a model-based SMC for space robots to rendezvous and synchronize with a satellite. However, for the on-board computer, the model-based decoupling or coordination control strategies usually need calculating the model parameters that may lead to a high computational cost. Zhang et al. [26] proposed a TDE-based SMC (TDE-SMC) to achieve the adaptive robust decoupling of multi-arm space robots, in which TDE is adopted to achieve the model-free decoupling control. Considering the limited computing ability of the onboard computer and continuous nonlinear disturbances in outer space, such as gravity-gradient torque, aerodynamic torque, and earth magnetic torque [27], it is necessary to design an efficient and robust decoupling controller.

SMC is one of the most effective robust control methods with the attractive feature of keeping the systems insensitive to disturbances and uncertainties [28]. However, the discontinuous switching input usually brings the undesirable chattering effect that cannot be accepted by actuators and harms the mechanical system. The classical boundary layer method uses the saturation function to replace the sign function in the switching law design, but it may degrade the control accuracy and robustness of SMC. For eliminating the chattering effect, Yu et al. [29] proposed a terminal sliding mode control (TSMC) to achieve the fast convergence with continuous finite-time control. Jin et al. [30] proposed a model-free controller based on TDE and TSMC with an integral sliding surface to realize the high-accuracy tracking control of robots. In comparison to the first-order SMC, the second-order SMC can effectively avoid the chattering problem by changing the robust switching mechanism [28]. The second-order SMC puts the switching term (namely, the sign function) into the second-order derivative of the sliding surface vector and the control law is a continuous integral of its derivative, so that the chattering problem can be eliminated [31]. Typical second-order SMC strategies include the sub-optimal algorithm [32], the twisting algorithm [33], and STC [34]. Among them, STC plays a special role because it only requires the knowledge of the sliding surface without considering its derivative. STC is widely used in industrial robots [35], aerial vehicles [36], space tethered robots [37], [38], and other mechatronic systems. Based on the above analysis, we aimed to efficiently decouple the base attitude and the manipulator of semi-floating space robots using the second-order SMC. The main contributions of this paper are listed as follows.

(1) The unit quaternion representation of the satellitemanipulator system dynamics in the joint space is deduced in this paper. This representation can avoid the representation singularity issue of using the Euler angles and the redundant parameters of using the rotation matrix in the base attitude control.

(2) An efficient robust controller, TDE-STC, is designed for decoupling semi-floating space robots with considering joint friction, environment disturbance, and system uncertainty factors. The TDE-STC stability is proven using the LMI theorem, and a loose requirement is derived to design parameters of the controller. The proposed method synthesizes the adaptability of TDE and the robustness of STC, which can achieve an highaccuracy attitude decoupling performance. Compared with model-based SMC methods, the proposed method realizes the decoupling control without the need for calculating model parameters and its control output is absolutely continuous and chatter-free, so it is more suitable for the on-board computer.

The rest of this paper is organized as follows. The base attitude kinematics and the system dynamics represented by the unit quaternion are established in Section II. Comparative analysis of the CTC and TDE decoupling strategies is conducted and the TDE-STC scheme is proposed in Section III. Simulation studies on testing and verifying the control performance of the proposed method are conducted in Section IV. The conclusions are provided in Section V.

\section{System Modelling}

The typical schematic diagram of a satellite-manipulator robot is shown in Fig. 1. The whole system consists of a satellite base (denoted as body 0) and a manipulator with $n$ bodies, where the base has 6 DoFs ( 3 for the base position and 3 for the base attitude) and the manipulator has $n$ DoFs. Thus, the space robot has $n+6$ DoFs in total. Some symbols are defined in Table I. Unless otherwise specified, vectors in this paper are defined in the inertial frame $\Sigma_{I}$. Basic assumptions of modelling and controlling are given as follows.

Assumption 1: The space robot is working in the microgravity environment, namely, $g=0$.

Assumption 2: The space robot system is equipped with high motor torque momentum and reaction wheels, which can provide enough power to regulate the base attitude.

Assumption 3: The space robot mainly executes the pointto-point motion task in which the joints of the manipulator change their rotation directions at a very low frequency. Overall, the discontinuous joint friction just occasionally exists in the model so that nonlinear disturbances and uncertainties meet the Lipschitz condition approximately.

\section{A. Base attitude kinematics}

As the Euler angles may suffer the representation singularity issue, the unit quaternion $\boldsymbol{Q}_{0}=\left\{\eta_{0}, \boldsymbol{\epsilon}_{0}^{T}\right\}$ is used to describe the base attitude. Compared with the rotation matrix representation [39], the unit quaternion has less parameters. The angular velocity of the base $\omega_{0}=\left[\omega_{0 x}, \omega_{0 y}, \omega_{0 z}\right]^{T} \in \mathbb{R}^{3}$ is defined 


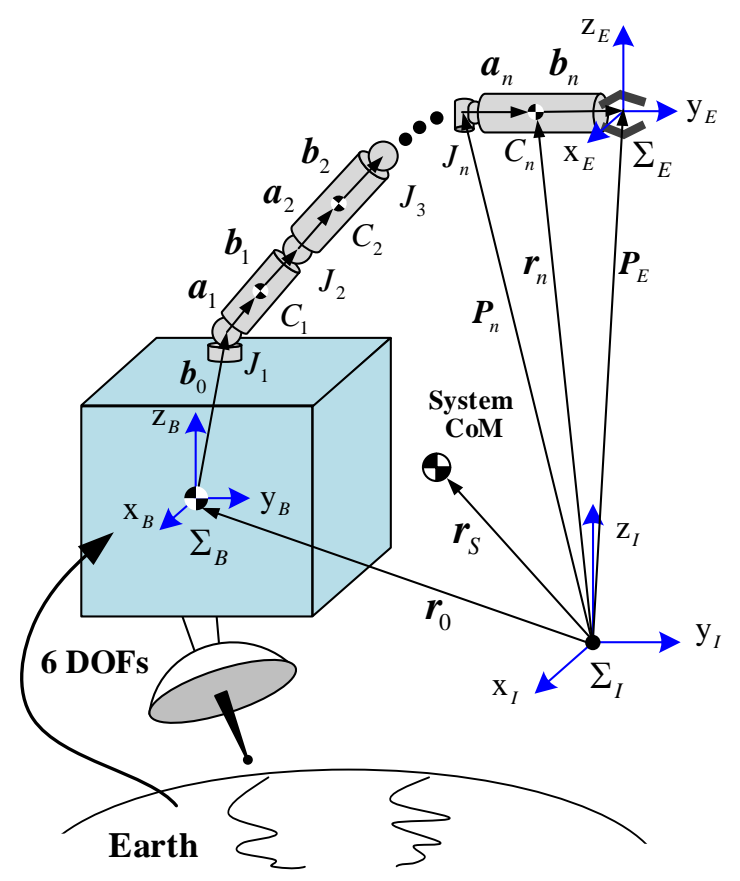

Fig. 1: Schematic diagram of a satellite-manipulator system

TABLE I: Symbol Definitions

\begin{tabular}{ll}
\hline Symbol & Definition \\
\hline$J_{i}$ & Joint $i$ \\
$C_{i}$ & Center of mass $(\mathrm{CoM})$ of the body $i$ \\
$\Sigma_{I}, \Sigma_{i}$ & Inertial and body $i$ frames \\
$\Sigma_{B}, \Sigma_{E}$ & Base and end-effector frames \\
$\mathrm{x}, \mathrm{y}, \mathrm{z}$ & Coordinates in the frame $\Sigma$ \\
$\theta_{i}, \boldsymbol{\theta} \in \mathbb{R}^{n}$ & Angle of $J_{i}$ and the joint angle vector \\
$\boldsymbol{z}_{i} \in \mathbb{R}^{3}$ & Unit vector of $J_{i}$ \\
$\boldsymbol{a}_{i} \in \mathbb{R}^{3}$ & Position vector from $J_{i}$ to $C_{i}$ \\
$\boldsymbol{b}_{i} \in \mathbb{R}^{3}$ & Position vector from $C_{i}$ to $J_{i+1}$ \\
$\boldsymbol{r}_{S} \in \mathbb{R}^{3}$ & Position vector of the system CoM \\
$\boldsymbol{r}_{0}, \boldsymbol{r}_{i} \in \mathbb{R}^{3}$ & Position vectors of $\Sigma_{B}$ and $C_{i}$ \\
$\boldsymbol{P}_{i}, \boldsymbol{P}_{E} \in \mathbb{R}^{3}$ & Position vectors of $J_{i}$ and $\Sigma_{E}$ \\
\hline
\end{tabular}

in $\Sigma_{B}$, and the kinematics equations of the base are deduced as follows.

$$
\begin{aligned}
\dot{\boldsymbol{\epsilon}}_{0}=\boldsymbol{J}_{0} \boldsymbol{\omega}_{0} & =\frac{1}{2}\left(\tilde{\boldsymbol{\epsilon}}_{0}+\eta_{0} \boldsymbol{E}\right) \boldsymbol{\omega}_{0} \\
\dot{\eta}_{0} & =-\frac{1}{2} \boldsymbol{\epsilon}_{0}^{T} \boldsymbol{\omega}_{0}
\end{aligned}
$$

where $\epsilon_{0}=\left[\epsilon_{0 x}, \epsilon_{0 y}, \epsilon_{0 z}\right]^{T} \in \mathbb{R}^{3}$ is the vector component; $\eta_{0}$ is the scalar component that satisfies the constraint condition, $\boldsymbol{\epsilon}_{0}^{T} \boldsymbol{\epsilon}_{0}+\eta_{0}^{2}=1 ; \boldsymbol{E} \in \mathbb{R}^{3 \times 3}$ is the identity matrix; thus, $\boldsymbol{Q}_{0}$ has three independent variables. The symbol $(*)$ denotes a skew-symmetric matrix, namely, for an arbitrary vector $\boldsymbol{k}=\left[k_{x}, k_{y}, k_{z}\right]^{T} \in \mathbb{R}^{3}, \tilde{\boldsymbol{k}}$ is defined as

$$
\tilde{\boldsymbol{k}}=\left[\begin{array}{ccc}
0 & -k_{z} & k_{y} \\
k_{z} & 0 & -k_{x} \\
-k_{y} & k_{x} & 0
\end{array}\right]
$$

\section{B. Satellite-manipulator system dynamics}

The dynamics model is deduced by the composite rigid dynamics modelling approach [40] that combines the Lagrange's and Newton-Euler methods, and it achieves a higher efficiency. Based on Assumption (1), the energy of the space robot system is mainly embodied in its kinetic energy. Here, the kinetic energy $\mathrm{T}$ is expressed as

$$
\begin{gathered}
\mathrm{T}=\frac{1}{2} \sum_{i=0}^{n}\left(\boldsymbol{\omega}_{i}^{T} \boldsymbol{I}_{i} \boldsymbol{\omega}_{i}+\boldsymbol{v}_{i}^{T} m_{i} \boldsymbol{v}_{i}\right) \\
\boldsymbol{\omega}_{i}=\boldsymbol{\omega}_{0}+\sum_{j=1}^{i} \boldsymbol{z}_{j} \dot{\theta}_{j} \\
\boldsymbol{v}_{i}=\boldsymbol{v}_{0}+\boldsymbol{\omega}_{0} \times\left(\boldsymbol{r}_{i}-\boldsymbol{r}_{0}\right)+\sum_{j=1}^{i}\left(\boldsymbol{z}_{j} \times\left(\boldsymbol{r}_{i}-\boldsymbol{P}_{j}\right)\right) \dot{\theta}_{j}
\end{gathered}
$$

where $m_{i}$ and $\boldsymbol{I}_{i} \in \mathbb{R}^{3}$ are the mass and inertial tensor of body $i$, respectively; $\boldsymbol{\omega}_{i} \in \mathbb{R}^{3}$ and $\boldsymbol{v}_{i} \in \mathbb{R}^{3}$ are the angular velocity of body $i$ and the linear velocity of the CoM of body $i$, respectively. Based on the Lagrange's principle, the general dynamics model of the space robot system is formulated as

$$
\underbrace{\left[\begin{array}{cc}
\boldsymbol{H}_{B} & \boldsymbol{H}_{B M} \\
\boldsymbol{H}_{B M}^{T} & \boldsymbol{H}_{M}
\end{array}\right]}_{\boldsymbol{H}(\boldsymbol{\rho})} \underbrace{\left[\begin{array}{c}
\ddot{\boldsymbol{x}}_{0} \\
\ddot{\boldsymbol{\theta}}
\end{array}\right]}_{\ddot{\boldsymbol{q}}}+\underbrace{\left[\begin{array}{c}
\boldsymbol{c}_{B} \\
\boldsymbol{c}_{M}
\end{array}\right]}_{\boldsymbol{c}(\boldsymbol{\rho}, \dot{\boldsymbol{q}})}=\underbrace{\left[\begin{array}{c}
\boldsymbol{F}_{B} \\
\boldsymbol{\tau}_{M}
\end{array}\right]}_{\boldsymbol{F}}
$$

where $\boldsymbol{H} \in \mathbb{R}^{(n+6) \times(n+6)}$ is the inertial matrix of the system; $\boldsymbol{H}_{B} \in \mathbb{R}^{6 \times 6}$ and $\boldsymbol{H}_{M} \in \mathbb{R}^{n \times n}$ are the inertial matrices of the base and the manipulator, respectively; $\boldsymbol{H}_{B M} \in \mathbb{R}^{n \times 6}$ is the coupling matrix between the base and the manipulator; $c \in \mathbb{R}^{n+6}$ is the velocity-dependent nonlinear term of the system; $\boldsymbol{c}_{B} \in \mathbb{R}^{6}$ and $\boldsymbol{c}_{M} \in \mathbb{R}^{n}$ are velocity-dependent nonlinear terms corresponding to the base and the manipulator, respectively; $\boldsymbol{F} \in \mathbb{R}^{n+6}$ is the driving force term of the system; $\boldsymbol{F}_{B}=\left[\boldsymbol{f}_{0}^{T}, \tau_{0}^{T}\right]^{T} \in \mathbb{R}^{6}$ are force and torque exerted on the base; $\tau_{M}=\left[\tau_{1}, \tau_{2}, \cdots, \tau_{n}\right]^{T} \in \mathbb{R}^{n}$ is the joint torque term of the manipulator; $\boldsymbol{\theta}=\left[\theta_{1}, \theta_{2}, \cdots, \theta_{n}\right]^{T} \in \mathbb{R}^{n}$ is the angle vector of the manipulator; $\ddot{\boldsymbol{x}}_{0}=\left[\dot{\boldsymbol{v}}_{0}^{T}, \dot{\boldsymbol{\omega}}_{0}^{T}\right]^{T} \in \mathbb{R}^{6}$ is the generalized acceleration of the base; $\dot{\boldsymbol{q}}$ and $\ddot{\boldsymbol{q}} \in \mathbb{R}^{n+6}$ are the generalized velocity and acceleration terms, respectively; and $\boldsymbol{\rho}=\left[\boldsymbol{r}_{0}^{T}, \boldsymbol{\epsilon}_{0}^{T}, \boldsymbol{\theta}^{T}\right]^{T} \in \mathbb{R}^{n+6}$ is the generalized position variable term. Here, note that $\rho$ cannot be obtained by the integral of $\dot{\boldsymbol{q}}$. Furthermore, by transforming (4) into

$$
\mathrm{T}=\frac{1}{2} \underbrace{\left[\begin{array}{lll}
\boldsymbol{v}_{0}^{T} & \boldsymbol{\omega}_{0}^{T} & \dot{\boldsymbol{\theta}}^{T}
\end{array}\right]}_{\dot{\boldsymbol{q}}^{T}} \underbrace{\left[\begin{array}{ccc}
M \boldsymbol{E} & -M \tilde{\boldsymbol{r}}_{0 S} & \boldsymbol{J}_{T \omega} \\
M \tilde{\boldsymbol{r}}_{0 S} & \boldsymbol{H}_{\omega} & \boldsymbol{H}_{\omega \phi} \\
\boldsymbol{J}_{T \omega}^{T} & \boldsymbol{H}_{\omega \phi}^{T} & \boldsymbol{H}_{M}
\end{array}\right]}_{\boldsymbol{H}(\boldsymbol{\rho})} \underbrace{\left[\begin{array}{c}
\boldsymbol{v}_{0} \\
\boldsymbol{\omega}_{0} \\
\dot{\boldsymbol{\theta}}
\end{array}\right]}_{\dot{\boldsymbol{q}}}
$$

we can deduce

$$
\boldsymbol{H}_{B}=\left[\begin{array}{cc}
M \boldsymbol{E} & -M \tilde{\boldsymbol{r}}_{0 S} \\
M \tilde{\boldsymbol{r}}_{0 S} & \boldsymbol{H}_{\omega}
\end{array}\right]
$$

$$
\boldsymbol{H}_{B M}=\left[\begin{array}{l}
\boldsymbol{J}_{T \omega} \\
\boldsymbol{H}_{\omega \phi}
\end{array}\right]
$$


and other parameters are expressed as

$$
\begin{gathered}
M=\sum_{i=0}^{n} m_{i} \\
\boldsymbol{r}_{0 S}=\boldsymbol{r}_{S}-\boldsymbol{r}_{0} \\
\boldsymbol{H}_{\omega}=\boldsymbol{I}_{0}+\sum_{i=1}^{n}\left(\boldsymbol{I}_{i}-m_{i} \tilde{\boldsymbol{r}}_{0 i} \tilde{\boldsymbol{r}}_{0 i}\right) \\
\boldsymbol{J}_{T \omega}=\sum_{i=1}^{n} m_{i} \boldsymbol{J}_{T i} \\
\boldsymbol{H}_{\omega \phi}=\sum_{i=1}^{n}\left(\boldsymbol{I}_{i} \boldsymbol{J}_{R i}+m_{i} \tilde{\boldsymbol{r}}_{0 i} \boldsymbol{J}_{T i}\right) \\
\boldsymbol{H}_{M}=\sum_{i=1}^{n}\left(\boldsymbol{J}_{R i}^{T} \boldsymbol{I}_{i} \boldsymbol{J}_{R i}+m_{i} \boldsymbol{J}_{T i}^{T} \boldsymbol{J}_{T i}\right)
\end{gathered}
$$

where

$$
\begin{gathered}
\boldsymbol{r}_{S}=\frac{\sum_{i=0}^{n} m_{i} \boldsymbol{r}_{i}}{M} \\
\boldsymbol{r}_{0 i}=\boldsymbol{r}_{i}-\boldsymbol{r}_{0} \\
\boldsymbol{J}_{T i}=\left[\boldsymbol{z}_{1} \times\left(\boldsymbol{r}_{i}-\boldsymbol{P}_{1}\right), \cdots, \boldsymbol{z}_{i} \times\left(\boldsymbol{r}_{i}-\boldsymbol{P}_{i}\right), \mathbf{0}, \cdots, \mathbf{0}\right] \\
\boldsymbol{J}_{R i}=\left[\boldsymbol{z}_{1}, \cdots, \boldsymbol{z}_{i}, \mathbf{0}, \cdots, \mathbf{0}\right]
\end{gathered}
$$

It should be noted that $\boldsymbol{r}_{i}, \boldsymbol{P}_{i}$, and $\boldsymbol{z}_{i}$ are defined in $\Sigma_{I}$, and they are functions of $\boldsymbol{\rho}$. Thus, $\boldsymbol{H}$ is a function of $\boldsymbol{\rho}$.

The term $c$ in (7) is called the velocity-dependent nonlinear term and not related to the system acceleration term. According to [40], $c$ can be easily obtained using the Newton-Euler method. Here, we can calculate $c$ by setting the generalized acceleration $\ddot{\boldsymbol{q}}$ to be $\mathbf{0}$, and the recursive steps include two parts, namely, the outward recursive computation of the joint velocity and acceleration vectors and the inward recursive computation of the joint force and torque vectors. First, we can reformulate (5) and (6) to deduce the velocity recursion equations as

$$
\begin{gathered}
\boldsymbol{\omega}_{i}=\boldsymbol{\omega}_{i-1}+\boldsymbol{z}_{i} \dot{\theta}_{i},(i=1,2, \cdots, n) \\
\boldsymbol{v}_{i}=\boldsymbol{v}_{i-1}+\boldsymbol{\omega}_{i-1} \times \boldsymbol{b}_{i-1}+\boldsymbol{\omega}_{i} \times \boldsymbol{a}_{i},(i=1,2, \cdots, n)
\end{gathered}
$$

Then, the acceleration recursion equations can be derived as

$$
\begin{gathered}
\dot{\boldsymbol{\omega}}_{i}=\dot{\boldsymbol{\omega}}_{i-1}+\boldsymbol{\omega}_{i-1} \times \boldsymbol{z}_{i} \dot{\theta}_{i}+\boldsymbol{z}_{i} \ddot{\theta}_{i},(i=1,2, \cdots, n) \\
\dot{\boldsymbol{v}}_{i}=\dot{\boldsymbol{v}}_{i-1}+\dot{\boldsymbol{\omega}}_{i-1} \times \boldsymbol{b}_{i-1}+\boldsymbol{\omega}_{i-1} \times\left(\boldsymbol{\omega}_{i-1} \times \boldsymbol{b}_{i-1}\right)+\dot{\boldsymbol{\omega}}_{i} \times \boldsymbol{a}_{i} \\
+\boldsymbol{\omega}_{i} \times\left(\boldsymbol{\omega}_{i} \times \boldsymbol{a}_{i}\right),(i=1,2, \cdots, n)
\end{gathered}
$$

Furthermore, the force and torque recursion equations can be deduced as

$$
\begin{gathered}
\boldsymbol{f}_{R i}=m_{i} \dot{\boldsymbol{v}}_{i} \\
\boldsymbol{n}_{R i}=\boldsymbol{I}_{i} \dot{\boldsymbol{\omega}}_{i}+\boldsymbol{\omega}_{i} \times \boldsymbol{I} \boldsymbol{\omega}_{i}
\end{gathered}
$$

where $\boldsymbol{f}_{R i} \in \mathbb{R}^{3}$ and $\boldsymbol{n}_{R i} \in \mathbb{R}^{3}$ are the resultant force and torque acting on body $i$, respectively. The following equations can be obtained by the force analysis.

$$
\begin{gathered}
\boldsymbol{f}_{i}=\boldsymbol{f}_{R i}+\boldsymbol{f}_{i+1},(i=n-1, n-2, \cdots, 0) \\
\boldsymbol{n}_{i}=\boldsymbol{n}_{R i}+\boldsymbol{n}_{i+1}-\boldsymbol{f}_{i} \times \boldsymbol{a}_{i}+\boldsymbol{f}_{i+1} \times \boldsymbol{b}_{i},(i=n-1, n-2, \cdots, 0)
\end{gathered}
$$

where, for the base, $\boldsymbol{f}_{0} \in \mathbb{R}^{3}$ and $\boldsymbol{\tau}_{0}=\boldsymbol{n}_{0} \in \mathbb{R}^{3}$ are the driving force and torque terms; for the manipulator, $\boldsymbol{f}_{i} \in \mathbb{R}^{3}$ and $\boldsymbol{n}_{i} \in$ $\mathbb{R}^{3}$ are the interaction force and torque between body $i-1$ and body $i$, respectively, and when $i=n$, we obtain $f_{n}=f_{R n}$ and $\boldsymbol{n}_{n}=\boldsymbol{n}_{R n}+\boldsymbol{n}_{n} \times \boldsymbol{a}_{n}$. Here, the driving torque of $J_{i}$ is expressed as

$$
\tau_{i}=\boldsymbol{n}_{i}^{T} \boldsymbol{z}_{i}
$$

According to (11)-(19), we can calculate the nonlinear term by setting $\ddot{\boldsymbol{q}}=\mathbf{0}$, and for simplicity, we denote

$$
c=\boldsymbol{f}_{N E}(\ddot{\boldsymbol{q}}=\mathbf{0})
$$

where $\boldsymbol{f}_{N E}(\cdot)$ is the Newton-Euler function. Moreover, the dynamic equation (7) can be written in a concise form as

$$
H(\rho) \ddot{q}+c(\rho, \dot{q})=F
$$

As shown in (21), $\rho$ is used to represent the position state variable term, and $\dot{\boldsymbol{q}}$ and $\ddot{\boldsymbol{q}}$ are used to represent the velocity and acceleration state variable terms, respectively. However, $\rho$ cannot be obtained by the integral of $\dot{\boldsymbol{q}}$. Therefore, we should perform some coordinate transformations to unify the state variables. According to (1), we can deduce

$$
\begin{gathered}
\dot{\boldsymbol{\rho}}=\boldsymbol{J} \dot{\boldsymbol{q}} \Rightarrow \dot{\boldsymbol{q}}=\boldsymbol{J}^{-1} \dot{\boldsymbol{\rho}} \\
\boldsymbol{J}=\left[\begin{array}{ccc}
\boldsymbol{E} & \mathbf{0} & \mathbf{0} \\
\mathbf{0} & \boldsymbol{J}_{0} & \mathbf{0} \\
\mathbf{0} & \mathbf{0} & \boldsymbol{E}_{n \times n}
\end{array}\right] \\
\ddot{\boldsymbol{\rho}}=\boldsymbol{J} \ddot{\boldsymbol{q}}+\dot{\boldsymbol{J}} \dot{\boldsymbol{q}} \Rightarrow \ddot{\boldsymbol{q}}=\boldsymbol{J}^{-1}\left(\ddot{\boldsymbol{\rho}}-\dot{\boldsymbol{J}} \boldsymbol{J}^{-1} \dot{\boldsymbol{\rho}}\right)
\end{gathered}
$$

Furthermore, substituting (22) and (24) into (21) and premultiplying the result with $\boldsymbol{J}^{-T}$ to ensure the transformed inertial matrix to be positive definite, we can obtain the standard rigid body dynamics equation as

$$
H_{S}(\rho) \ddot{\rho}+c_{S}(\rho, \dot{\rho})=\boldsymbol{F}
$$

where

$$
\begin{gathered}
\boldsymbol{H}_{S}(\boldsymbol{\rho})=\boldsymbol{J}^{-T} \boldsymbol{H}(\boldsymbol{\rho}) \boldsymbol{J}^{-1} \\
\boldsymbol{c}_{S}(\boldsymbol{\rho}, \dot{\boldsymbol{\rho}})=\boldsymbol{J}^{-T}\left(\boldsymbol{c}(\boldsymbol{\rho}, \dot{\boldsymbol{q}})-\boldsymbol{H}(\boldsymbol{\rho}) \boldsymbol{J}^{-1} \dot{\boldsymbol{J}} \boldsymbol{J}^{-1} \dot{\boldsymbol{\rho}}\right)
\end{gathered}
$$

and in $\dot{\boldsymbol{J}}, \dot{\boldsymbol{J}}_{0} \in \mathbb{R}^{3 \times 3}$ is expressed as

$$
\dot{\boldsymbol{J}}_{0}=\frac{1}{2}\left(\dot{\tilde{\boldsymbol{\epsilon}}}_{0}+\dot{\eta}_{0} \boldsymbol{E}\right)
$$

For the nominal model (25), it is nonlinear and continuous. Sometimes, we should consider the joint friction which is usually assumed to be composed of viscous and Coulomb friction terms. When joint friction, external disturbances, and 
parameter uncertainties are considered in the nominal model, (25) is modified to

$$
\boldsymbol{H}_{S}(\boldsymbol{\rho}) \ddot{\boldsymbol{\rho}}+c_{S}(\boldsymbol{\rho}, \dot{\boldsymbol{\rho}})+\boldsymbol{f}_{d}+\boldsymbol{f}_{u}+\boldsymbol{f}_{f}=\boldsymbol{F}
$$

where $\boldsymbol{f}_{f}=\left[\mathbf{0}, \boldsymbol{f}_{M}\right]^{T} \in \mathbb{R}^{n+6}$ is the friction torque and $\boldsymbol{f}_{M} \in \mathbb{R}^{n}$ is the manipulator friction force vector described by $\boldsymbol{f}_{M}=$ $\boldsymbol{f}_{C} \operatorname{sign}(\dot{\boldsymbol{\theta}})+\boldsymbol{f}_{V} \dot{\boldsymbol{\theta}}$, where $\boldsymbol{f}_{C}=\operatorname{diag}\left(f_{C 1}, f_{C 2}, \cdots, f_{C n}\right) \in \mathbb{R}^{n \times n}$ is the Coulomb friction parameter matrix and each parameter is restricted by its boundedness, namely, $\left|f_{C i}\right| \leq C_{i}$ and $\boldsymbol{f}_{V}=$ $\operatorname{diag}\left(f_{V 1}, f_{V 2}, \cdots, f_{V n}\right) \in \mathbb{R}^{n \times n}$ is the viscous friction parameter matrix and each parameter is restricted by its boundedness, namely, $\left|f_{V i}\right| \leq V_{i} ; \boldsymbol{f}_{d}=\left[f_{d 1}, f_{d 2}, \cdots, f_{d(n+6)}\right]^{T} \in \mathbb{R}^{n+6}$ is the disturbance force and each element is restricted by its boundedness, i.e., $\left|f_{d i}\right| \leq d_{i} ; \boldsymbol{f}_{u}=\left[f_{u 1}, f_{u 2}, \cdots, f_{u(n+6)}\right]^{T} \in \mathbb{R}^{n+6}$ describes uncertainty dynamics and each element is restricted by its boundedness, namely, $\left|f_{u i}\right| \leq u_{i}$. As we only seek to achieve the base attitude decoupling control, the base position is not under control. Thus, we can deduce

$$
\boldsymbol{H}_{S}(\boldsymbol{\rho}) \ddot{\boldsymbol{\rho}}+\boldsymbol{c}_{S}(\boldsymbol{\rho}, \dot{\boldsymbol{\rho}})+\boldsymbol{f}_{d}+\boldsymbol{f}_{u}+\boldsymbol{f}_{f}=\boldsymbol{F}_{S}
$$

where $\boldsymbol{F}_{S}=\boldsymbol{D}_{S} \boldsymbol{F}=\left[0,0,0, F_{S 4}, \cdots, F_{S(n+6)}\right]^{T} \in \mathbb{R}^{n+6}$ is the driving torque for the semi-floating space robot, and the base attitude decoupling matrix $D_{S}$ is expressed as

$$
\boldsymbol{D}_{S}=\left[\begin{array}{cc}
\mathbf{0}_{3 \times 3} & \mathbf{0}_{3 \times(n+3)} \\
\mathbf{0}_{(n+3) \times 3} & \boldsymbol{E}_{(n+3) \times(n+3)}
\end{array}\right]
$$

\section{Decoupling controllers}

In this section, we will discuss and analyze the CTC and TDE decoupling strategies. Since TDE has the model-free merits, we seek to use the TDE principle to address the attitude coupling issue of semi-floating space robots. Considering that STC has the chatter-free advantages, an effective robust controller is proposed by combining TDE and STC. Meanwhile, several existing decoupling or coordination control methods will be introduced for comparative studies.

\section{A. Decoupling strategies}

CTC is the most widely used model-based decoupling strategy that requires the calculation of the model parameters such as the inertial matrix and the nonlinear term to realize the dynamics decoupling, so it is an online computation method [41]. TDE is a model-free decoupling control strategy [42], which uses the current feedback information and the control input at the previous time to estimate the coupled dynamics, so it is an online estimation method. Next, we will compare these two decoupling control strategies based on their principles.

The CTC control law is expressed as

$$
\begin{gathered}
\boldsymbol{F}=\boldsymbol{H}_{S} \boldsymbol{u}_{P D}+\boldsymbol{c}_{S} \\
\boldsymbol{u}_{P D}=\ddot{\boldsymbol{\rho}}_{d}+\boldsymbol{K}_{D}\left(\dot{\boldsymbol{\rho}}_{d}-\dot{\boldsymbol{\rho}}\right)+\boldsymbol{K}_{P}\left(\boldsymbol{\rho}_{d}-\boldsymbol{\rho}\right) \\
=\ddot{\boldsymbol{\rho}}_{d}+\boldsymbol{K}_{D} \dot{\boldsymbol{e}}+\boldsymbol{K}_{P} \boldsymbol{e}
\end{gathered}
$$

where $\boldsymbol{u}_{P D} \in \mathbb{R}^{n+6}$ is the PD control with a bias term $\ddot{\boldsymbol{\rho}}_{d}$; $\boldsymbol{K}_{P} \in \mathbb{R}^{(n+6) \times(n+6)}$ and $\boldsymbol{K}_{D} \in \mathbb{R}^{(n+6) \times(n+6)}$ are proportional and derivative gain matrices, respectively; and $e \in \mathbb{R}^{n+6}$ and $\dot{e} \in \mathbb{R}^{n+6}$ are the state error and its derivative, respectively.
Substituting (30) into (25), we can obtain a completely decoupled linear model:

$$
\boldsymbol{H}_{S} \ddot{\boldsymbol{\rho}}=\boldsymbol{H}_{S} \boldsymbol{u}_{P D} \Rightarrow \ddot{\boldsymbol{\rho}}=\boldsymbol{u}_{P D}
$$

As we see, the CTC scheme is more suitable for an accurate model. Next, we will introduce the TDE decoupling control strategy.

Lemma 1: For $\boldsymbol{H}_{S}$, it is always feasible to find a constant diagonal matrix $\overline{\boldsymbol{H}}$ that meets the spectral norm condition, $\left\|\boldsymbol{E}-\boldsymbol{H}_{S}^{-1}(t) \overline{\boldsymbol{H}}\right\|<1$ [43].

For TDE, a constant diagonal matrix $\overline{\boldsymbol{H}} \in \mathbb{R}^{(n+6) \times(n+6)}$ from Lemma 1 is added into (27) to reformat the dynamics model. The new model is formulated as

$$
\begin{gathered}
\overline{\boldsymbol{H}} \ddot{\boldsymbol{\rho}}+\boldsymbol{N}=\boldsymbol{F} \\
\boldsymbol{N}=\left(\boldsymbol{H}_{S}-\overline{\boldsymbol{H}}\right) \ddot{\boldsymbol{\rho}}+\boldsymbol{c}_{S}+\boldsymbol{f}_{f}+\boldsymbol{f}_{d}+\boldsymbol{f}_{u}
\end{gathered}
$$

where $N \in \mathbb{R}^{n+6}$ is the new nonlinear term which includes the coupled inertial term and nonlinear terms. According to (33), $\overline{\boldsymbol{H}}$ is the constant diagonal matrix; if $\boldsymbol{N}$ can be accurately estimated, (33) can be decoupled and linearized. According to the TDE principle, we can estimate $N$ via the time-delay information, as

$$
\mathbf{N}(t) \approx \hat{\mathbf{N}}(t)=\mathbf{N}(t-\delta)
$$

where $\hat{N}(t)$ is the TDE term representing the time-delay estimation of $\boldsymbol{N}(t)$ and $\delta$ is the sampling period. The core part of the TDE technique is (35). According to (33), we can obtain

$$
\boldsymbol{N}(t-\delta)=\boldsymbol{F}(t-\delta)-\overline{\boldsymbol{H}} \ddot{\boldsymbol{\rho}}(t-\delta)
$$

The TDE control law is expressed as

$$
\boldsymbol{F}(t)=\overline{\boldsymbol{H}} \boldsymbol{u}_{P D}(t)+\hat{\boldsymbol{N}}(t)
$$

where $\boldsymbol{u}_{P D}(t)$ is the same as (31). As we see, the technical approaches of CTC and TDE are completely different. CTC must calculate the model parameters to design the control law, but TDE utilizes the time-delay feedback and control input information to estimate the coupled dynamics, which suits the inaccurate model. TDE is more efficient and effective and has low computational complexity, but the estimation error (namely, the TDE error) is its intrinsic drawback. According to (27), the nonlinear terms in space robot dynamics are classified into two categories: the soft nonlinearities (including the coupled inertial term, the velocity dependent nonlinear term, the viscous friction, continuous disturbances and uncertain dynamics) and the hard nonlinearities (such as the Coulomb friction term). TDE has a good application effect to estimate the soft nonlinearities [44]. However, it is difficult for TDE to estimate the hard nonlinearity. Fortunately, to a certain degree, SMC can reinforce TDE and suppress the bounded TDE error [45] . Herein, STC is adopted in the TDE-based controller, which can realize the high-accuracy decoupling control with the chatter-free merits. 


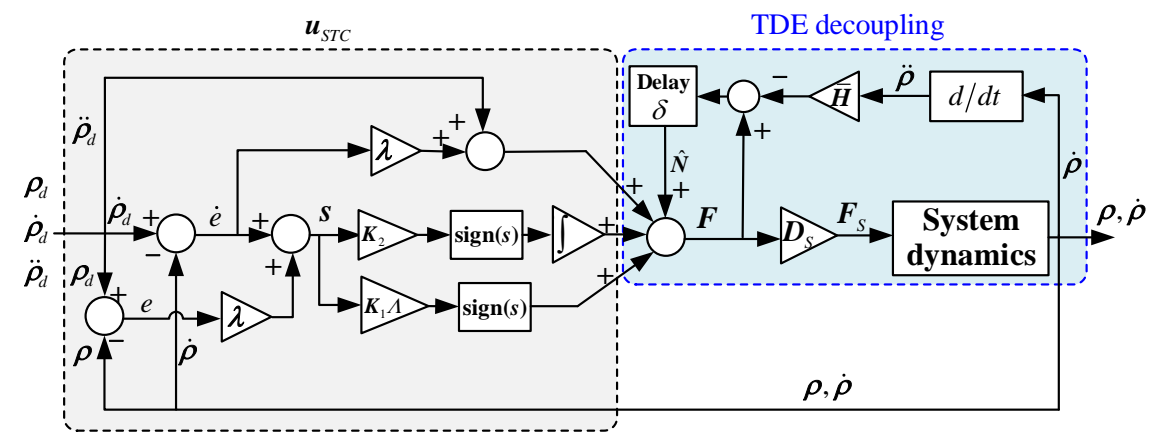

Fig. 2: TDE-STC

\section{B. Controller design}

SMC is a classical robust control in the presence of bounded disturbances and uncertainties, but the discontinuous highfrequency switching control usually leads to the chattering effect. However, STC is an absolutely continuous control algorithm, which has the following advantages:

(1) Its control law only requires the information of the sliding surface vector $s$ without considering its derivative $\dot{\boldsymbol{s}}$.

(2) It drives both $s$ and $\dot{s} \rightarrow \mathbf{0}$ simultaneously and in finite time.

(3) It generates the continuous control signal with the chatter-free merits.

Our objective is to design an effective and efficient controller by combing TDE and STC to realize the high-accuracy attitude decoupling control of the semi-floating space robot.

The principle of TDE-STC is shown in Fig. 2. First, a linear sliding surface is designed as

$$
s=\dot{e}+\lambda e
$$

where $\boldsymbol{\lambda}=\operatorname{diag}\left(\lambda_{1}, \lambda_{2}, \cdots, \lambda_{n+6}\right) \in \mathbb{R}^{(n+6) \times(n+6)}$ is positive definite. Furthermore, we can obtain

$$
\dot{s}=\ddot{e}+\lambda \dot{e}
$$

The STC algorithm is formulated as

$$
\left\{\begin{array}{c}
\dot{s}=-K_{1} \Lambda(s) \operatorname{sign}(s)+\psi \\
\dot{\psi}=-K_{2} \operatorname{sign}(s)
\end{array}\right.
$$

where $\Lambda(s)=\operatorname{diag}\left(\left|s_{1}\right|^{1 / 2},\left|s_{2}\right|^{1 / 2}, \cdots,\left|s_{n+6}\right|^{1 / 2}\right) \quad \in$ $\mathbb{R}^{(n+6) \times(n+6)} ; \operatorname{sign}(\cdot) \quad$ represents the signum function; $\boldsymbol{K}_{1}=\operatorname{diag}\left(K_{11}, K_{12}, \cdots, K_{1(n+6)}\right) \in \mathbb{R}^{(n+6) \times(n+6)}$ and $\boldsymbol{K}_{2}=\operatorname{diag}\left(K_{21}, K_{22}, \cdots, K_{2(n+6)}\right) \in \mathbb{R}^{(n+6) \times(n+6)}$ are positivedefinite matrices, respectively.

As TDE uses $\hat{N}$ to estimate $N$, the control law of TDESTC is expressed as

$$
\boldsymbol{F}=\overline{\boldsymbol{H}} \boldsymbol{u}_{S T C}+\hat{\boldsymbol{N}}
$$

$$
\boldsymbol{u}_{S T C}=\ddot{\boldsymbol{\rho}}_{d}+\lambda \dot{\boldsymbol{e}}+\boldsymbol{K}_{1} \Lambda(s) \operatorname{sign}(s)+\int_{0}^{t} \boldsymbol{K}_{2} \operatorname{sign}(s) d t
$$

Substituting (41) and (42) into (33), we can obtain the following closed-loop system.

$$
\begin{aligned}
\overline{\boldsymbol{H}}^{-1}(\boldsymbol{N}-\hat{\boldsymbol{N}})= & \ddot{\boldsymbol{\rho}}_{d}-\ddot{\boldsymbol{\rho}}+\lambda \dot{\boldsymbol{e}}+\boldsymbol{K}_{1} \Lambda(\boldsymbol{s}) \operatorname{sign}(\boldsymbol{s}) \\
& +\int_{0}^{t} \boldsymbol{K}_{2} \operatorname{sign}(s) d t
\end{aligned}
$$

Furthermore, according to (39), (43) can be reformulated as

$$
\varepsilon=\dot{s}+K_{1} \Lambda(s) \operatorname{sign}(s)+\int_{0}^{t} \boldsymbol{K}_{2} \operatorname{sign}(s) d t
$$

where $\varepsilon=\overline{\boldsymbol{H}}^{-1}(\boldsymbol{N}-\hat{\boldsymbol{N}}) \in \mathbb{R}^{(n+6)}$ is bounded and the corresponding proof can be referred to the boundedness of the TDE error [45]. Meanwhile, the closed-loop system (44) can be rewritten as

$$
\left\{\begin{array}{c}
\dot{s}=-K_{1} \Lambda(s) \operatorname{sign}(s)+\psi \\
\dot{\psi}=-K_{2} \operatorname{sign}(s)+\dot{\varepsilon}
\end{array}\right.
$$

where $\dot{\varepsilon}=\left[\dot{\varepsilon}_{1}, \cdots, \dot{\varepsilon}_{i}, \cdots, \dot{\varepsilon}_{(n+6)}\right]^{T} \in \mathbb{R}^{(n+6)}$ is the derivative of $\varepsilon$, and each element is restricted by its boundedness, namely, $\left|\dot{\varepsilon}_{i}\right| \leq \beta_{i}$. The proof of the boundedness of $\dot{\varepsilon}_{i}$ is presented in Appendix A. For the $i$ th closed-loop subsystem, we can obtain

$$
\left\{\begin{array}{c}
\dot{s}_{i}=-K_{1 i}\left|s_{i}\right|^{1 / 2} \operatorname{sign}\left(s_{i}\right)+\psi_{i} \\
\dot{\psi}_{i}=-K_{2 i} \operatorname{sign}\left(s_{i}\right)+\dot{\varepsilon}_{i}
\end{array}\right.
$$

when $K_{2 i}>\beta_{i}$ and $K_{1 i}^{2}>4 K_{2 i}$, the closed-loop system is stable.

For tuning the control parameters, some simple rules are described as follows: First, $\overline{\boldsymbol{H}}$ is a positive diagonal matrix and we gradually increase the diagonal elements while checking the convergence of the result. Then, we turn $\boldsymbol{K}_{1}$ and $\boldsymbol{K}_{2}$ while checking the control performance. As $\boldsymbol{K}_{2}$ is in the integral sign, we turn $\boldsymbol{K}_{2}$ from a small order of magnitude by the trial-and-error method.

\section{Stability and finite-time convergence analysis}

The stability of TDE-STC is analyzed by the LMI theorem which is similar in [46]. First, the Lyapunov function is defined as follows.

$$
V=\boldsymbol{\eta}^{T} \boldsymbol{M} \boldsymbol{\eta}
$$


where $\boldsymbol{\eta}=\left[\eta_{1}, \eta_{2}\right]^{T}=\left[\left|s_{i}\right|^{1 / 2} \operatorname{sign}\left(s_{i}\right), \psi_{i}\right]^{T} \in \mathbb{R}^{2}$ and $\boldsymbol{M} \in$ $\mathbb{R}^{2 \times 2}$ is a symmetric positive definite matrix expressed as

$$
M=\frac{1}{2}\left[\begin{array}{cc}
K_{1 i}^{2}+4 K_{2 i} & -K_{1 i} \\
-K_{1 i} & 2
\end{array}\right]
$$

The derivative of $\boldsymbol{\eta}$ is expressed as

$$
\dot{\eta}=\left[\begin{array}{c}
\frac{1}{2\left|s_{i}\right|^{1 / 2}} \dot{s}_{i} \\
\dot{\psi}_{i}
\end{array}\right]
$$

Meanwhile, we know that $\left|\eta_{1}\right|=\left|s_{i}\right|^{1 / 2}$. According to (46), $\dot{\eta}$ can be rewritten as

$$
\dot{\boldsymbol{\eta}}=\frac{1}{\left|\eta_{1}\right|}(\boldsymbol{A} \boldsymbol{\eta}+\boldsymbol{B} \varphi)
$$

where

$$
\boldsymbol{A}=\left[\begin{array}{cc}
-\frac{1}{2} K_{1 i} & \frac{1}{2} \\
-K_{2 i} & 0
\end{array}\right], \boldsymbol{B}=\left[\begin{array}{l}
0 \\
1
\end{array}\right], \varphi=\dot{\varepsilon}_{i}\left|\eta_{1}\right|
$$

There exists $|\varphi| \leq \beta_{i}\left|\eta_{1}\right|$ and we can deduce $\phi=-\varphi^{2}+$ $\beta_{i}^{2}\left|\eta_{1}\right|^{2} \geq 0$

Lemma 2: There always exists a symmetrical positive definite matrix $M$ that makes the following LMI hold [47].

$$
\left[\begin{array}{cc}
\boldsymbol{A}^{T} \boldsymbol{M}+\boldsymbol{M A}+\gamma \boldsymbol{M}+\beta_{i}^{2} \boldsymbol{C}^{T} \boldsymbol{C} & \boldsymbol{M B} \\
\boldsymbol{B}^{T} \boldsymbol{M} & -1
\end{array}\right] \leq 0
$$

where $C=[1,0], \gamma>0$, and $K_{1 i}$ and $K_{2 i}$ meet the conditions: $K_{2 i}>\beta_{i}$ and $K_{1 i}^{2}>4 K_{2 i}$. The corresponding proof analyzed in the complex frequency domain is provided in Appendix B.

Furthermore, the derivative of $V$ is computed as

$$
\begin{aligned}
& \dot{V}=\dot{\boldsymbol{\eta}}^{T} \boldsymbol{M} \boldsymbol{\eta}+\boldsymbol{\eta}^{T} \boldsymbol{M} \dot{\boldsymbol{\eta}} \\
& =\frac{1}{\left|\eta_{1}\right|} \boldsymbol{\eta}^{T}\left(\boldsymbol{A}^{T} \boldsymbol{M}+\boldsymbol{M} \boldsymbol{A}\right) \boldsymbol{\eta}+\frac{2 \varphi}{\left|\eta_{1}\right|} \boldsymbol{B}^{T} \boldsymbol{M} \boldsymbol{\eta} \\
& =\frac{1}{\left|\eta_{1}\right|}\left[\begin{array}{l}
\boldsymbol{\eta} \\
\varphi
\end{array}\right]^{T}\left[\begin{array}{cc}
\boldsymbol{A}^{T} \boldsymbol{M}+\boldsymbol{M} \boldsymbol{A} & \boldsymbol{M} \boldsymbol{B} \\
\boldsymbol{B}^{T} \boldsymbol{M} & 0
\end{array}\right]\left[\begin{array}{l}
\boldsymbol{\eta} \\
\varphi
\end{array}\right] \\
& \leq \frac{1}{\left|\eta_{1}\right|}\left\{\left[\begin{array}{l}
\boldsymbol{\eta} \\
\varphi
\end{array}\right]^{T}\left[\begin{array}{cc}
\boldsymbol{A}^{T} \boldsymbol{M}+\boldsymbol{M} \boldsymbol{A} & \boldsymbol{M B} \\
\boldsymbol{B}^{T} \boldsymbol{M} & 0
\end{array}\right]\left[\begin{array}{l}
\boldsymbol{\eta} \\
\varphi
\end{array}\right]+\phi\right\} \\
& \leq \frac{1}{\left|\eta_{1}\right|}\left\{\left[\begin{array}{l}
\boldsymbol{\eta} \\
\varphi
\end{array}\right]^{T}\left[\begin{array}{cc}
\boldsymbol{A}^{T} \boldsymbol{M}+\underset{\boldsymbol{M} \boldsymbol{A}+\beta_{i}^{2} \boldsymbol{C}^{T} \boldsymbol{C}}{\boldsymbol{M B} \boldsymbol{B}} \\
\boldsymbol{B}^{T} \boldsymbol{M} & -1
\end{array}\right]\left[\begin{array}{l}
\boldsymbol{\eta} \\
\varphi
\end{array}\right]\right\} \\
& \leq \frac{1}{\left|\eta_{1}\right|}\left\{[ \begin{array} { l } 
{ \boldsymbol { \eta } } \\
{ \varphi }
\end{array} ] ^ { T } \left[\begin{array}{c}
\boldsymbol{A}^{T} \boldsymbol{M}+\boldsymbol{M A}+\gamma \boldsymbol{M}-\gamma \boldsymbol{M}+\beta_{i}^{2} \boldsymbol{C}^{T} \boldsymbol{C} \\
\boldsymbol{B}^{T} \boldsymbol{M}
\end{array}\right.\right. \\
& \leq \frac{-\gamma}{\left|\eta_{1}\right|} \boldsymbol{\eta}^{T} \boldsymbol{M} \boldsymbol{\eta}
\end{aligned}
$$

$\left.\left.\begin{array}{c}M B \\ -1\end{array}\right]\left[\begin{array}{l}\eta \\ \varphi\end{array}\right]\right\}$

Thus, we can deduce $\dot{V}$ is negative definite and the stability of TDE-STC is proven. The proof of its finite-time convergence [28] is presented as follows.

For the Lyapunov function (47), we can deduce

$$
\lambda_{\min }(\boldsymbol{M})\|\boldsymbol{\eta}\|^{2} \leq V \leq \lambda_{\max }(\boldsymbol{M})\|\boldsymbol{\eta}\|^{2}
$$

where $\lambda(\boldsymbol{M})$ represents the eigenvalue of $\boldsymbol{M}$ and $\|\boldsymbol{\eta}\|=$ $\sqrt{\eta_{1}^{2}+\eta_{2}^{2}}$ is the Euclidian norm of $\boldsymbol{\eta}$. Also, we can deduce $\left|\eta_{1}\right| \leq\|\eta\| \leq \frac{V^{1 / 2}}{\lambda_{\min }^{1 / 2}(\boldsymbol{M})}$. According to (52) and (47), we obtain

$$
\dot{V} \leq \frac{-\gamma}{\left|\eta_{1}\right|} V \leq-\gamma \lambda_{\text {min }}^{1 / 2}(M) V^{1 / 2}
$$

Furthermore, we set $\Psi=\gamma \lambda_{\text {min }}^{1 / 2}(M)$, so we can deduce $\dot{V}(\boldsymbol{\eta}) \leq-\Psi V^{1 / 2}(\boldsymbol{\eta})$. For $\dot{V}(\boldsymbol{\eta})=-\Psi V(\boldsymbol{\eta})^{1 / 2}$, integrating both sides with respect to time, we can deduce

$$
V^{1 / 2}(\boldsymbol{\eta}(t))-V^{1 / 2}(\boldsymbol{\eta}(0))=\frac{-\Psi}{2} t
$$

There exists a certain time $t=t_{r}$ so that $V\left(\boldsymbol{\eta}\left(t_{r}\right)\right)=0$. This is given by

$$
t_{r}=\frac{2}{\Psi} V^{1 / 2}(\boldsymbol{\eta}(0))
$$

Thus, the control law (41) drives $s$ and $\boldsymbol{\psi} \rightarrow \mathbf{0}$ in finite time. For the closed-loop system (45), the control law drives $\dot{\boldsymbol{s}} \rightarrow \mathbf{0}$ as well.

\section{Contrastive controllers}

In the following comparative studies, several decoupling controllers including CTC, CTC-SMC, TDE-SMC, and TDETSMC are introduced as follows. As we aim to control the semi-floating space robot, only the base attitude and manipulator control parts in the concurrent controllers [5], [24] are used in this paper. The corresponding concurrent control law in [5] is adopted, which is the same as the CTC control law (30), $\boldsymbol{F}=\boldsymbol{H}_{S} \boldsymbol{u}_{P D}+\boldsymbol{c}_{S}$.

The control law of CTC-SMC [22] with the boundary layer function is expressed as

$$
\boldsymbol{F}=\boldsymbol{H}_{S}\left(\ddot{\boldsymbol{\rho}}_{d}+\lambda \dot{e}\right)+\boldsymbol{c}_{S}+\boldsymbol{g} \operatorname{sat}(\boldsymbol{s}, \boldsymbol{\Delta})
$$

where $\boldsymbol{g}=\operatorname{diag}\left(g_{1}, g_{2}, \cdots, g_{(n+6)}\right) \in \mathbb{R}^{(n+6) \times(n+6)}$ is the robust switching gain matrix; $\boldsymbol{\Delta}=\left[\Delta_{1}, \Delta_{2}, \cdots, \Delta_{(n+6)}\right] \in \mathbb{R}^{(n+6)}$ is the boundary layer width vector; $\operatorname{sat}(s, \Delta)$ is the boundary layer function and its $i$ th element is defined as

$$
\operatorname{sat}\left(s_{i}, \Delta_{i}\right)=\left\{\begin{array}{l}
\frac{s_{i}}{\left|s_{i}\right|}, \text { if }\left|s_{i}\right|>\Delta_{i} \\
\frac{s_{i}}{\Delta_{i}}, \text { if }\left|s_{i}\right| \leq \Delta_{i}
\end{array}\right.
$$

The control law of TDE-SMC [26] is formulated as

$$
\boldsymbol{F}=\overline{\boldsymbol{H}}\left(\ddot{\boldsymbol{\rho}}_{d}+\lambda \dot{e}\right)+\hat{\boldsymbol{N}}+\boldsymbol{g} \operatorname{sign}(s)
$$

The control law of TDE-TSMC [30] is expressed as

$$
\boldsymbol{F}=\overline{\boldsymbol{H}}\left(\boldsymbol{u}_{P D}+\boldsymbol{K}_{1} \boldsymbol{\Lambda}(s) \operatorname{sign}(s)\right)+\hat{\boldsymbol{N}}
$$

where $\boldsymbol{u}_{P D}$ is the same as (31); $\boldsymbol{K}_{1} \boldsymbol{\Lambda}(\boldsymbol{s}) \operatorname{sign}(\boldsymbol{s})$ is the same as (40). Specially, $s$ is adopted an integral sliding surface, namely, $\boldsymbol{s}=\int_{0}^{t}\left(\ddot{\boldsymbol{e}}+\boldsymbol{K}_{D} \dot{\boldsymbol{e}}+\boldsymbol{K}_{P} \boldsymbol{e}\right) d t$.

For the four contrastive controllers above, the detailed parameter selection is described in [5], [22], [26], and [30], respectively.

\section{Simulation AND comparison}

In this section, we will verify the proposed control method using a 3-D satellite-manipulator system as shown in Fig. 3. The $\mathrm{D}-\mathrm{H}$ and inertial parameters of the satellite-manipulator system are given in Tables II and III, respectively. Here, a monitoring mission with and without the payload will be simulated to compare the performance of CTC, CTC-SMC, TDE-SMC, TDE-TSMC, and the proposed TDE-STC. As shown in Fig. 4, the mission includes two processes, namely, the manipulator from the initial configuration reaches the 


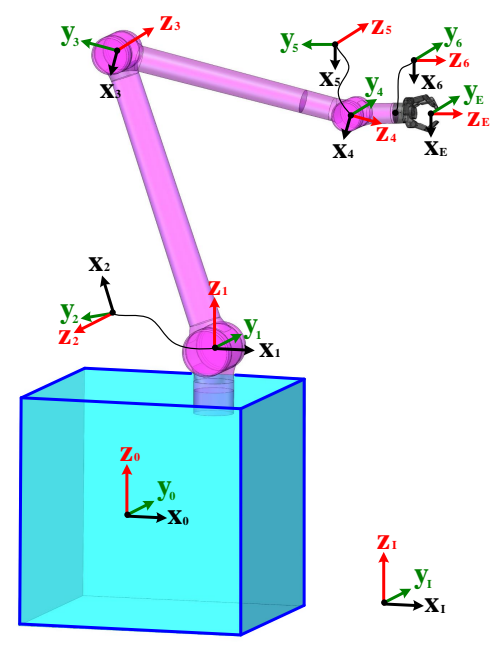

Fig. 3: Satellite-manipulator system

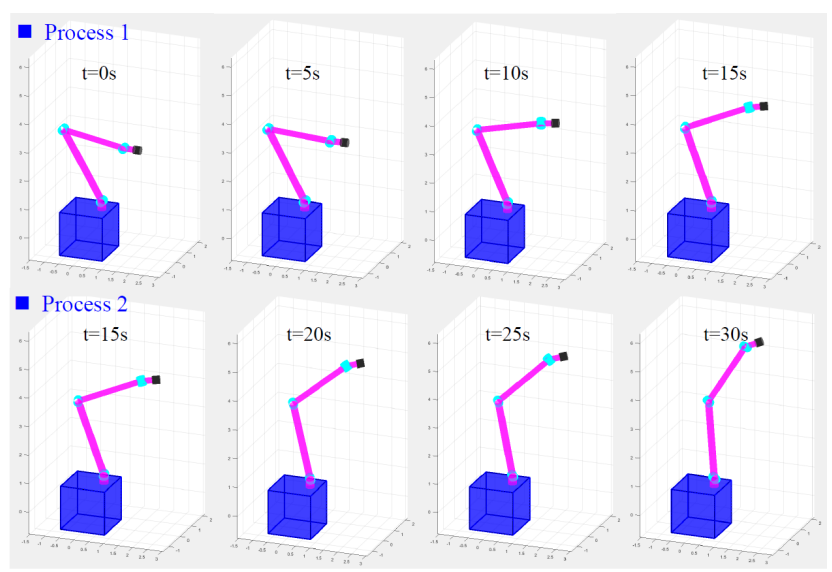

Fig. 4: Motion processes of the monitoring mission

middle configuration and then from the middle configuration to the final configuration. The base attitude is set as $\boldsymbol{Q}_{0}=\{1, \mathbf{0}\}$. Here, the middle configuration is set as $\boldsymbol{\theta}\left(t_{m}\right)=$ $[15,110,185,45,10,-25]^{T}(\mathrm{deg})$; the final configuration is set as $\boldsymbol{\theta}\left(t_{f}\right)=[30,100,150,90,30,-50]^{T}($ deg $)$; the middle time is set as $t_{m}=15 \mathrm{~s}$, and the final time is set as $t_{f}=30 \mathrm{~s}$; the middle monitoring state is set as $\dot{\boldsymbol{\theta}}\left(t_{m}\right)=\ddot{\boldsymbol{\theta}}\left(t_{m}\right)=\mathbf{0}$; the final monitoring state is set as $\dot{\boldsymbol{\theta}}\left(t_{f}\right)=\ddot{\boldsymbol{\theta}}\left(t_{f}\right)=\mathbf{0}$; and the fifthorder polynomial segmented functions are used to plan the joint trajectories (in Fig. 5). We conducted four case studies where the first three cases are studied to compare five different controllers and the last case is studied to verify the robustness of the proposed TDE-STC under consideration of different disturbances and model uncertainties. For the three TDE-based controllers, the sampling period is set as: $\delta=0.5 \mathrm{~ms}$. Control parameters of the five controllers are listed in Table IV. Since the base position is not controlled, the first three elements of the control parameters are set to be 0 and their control parameters are kept unchanged in the following case studies.

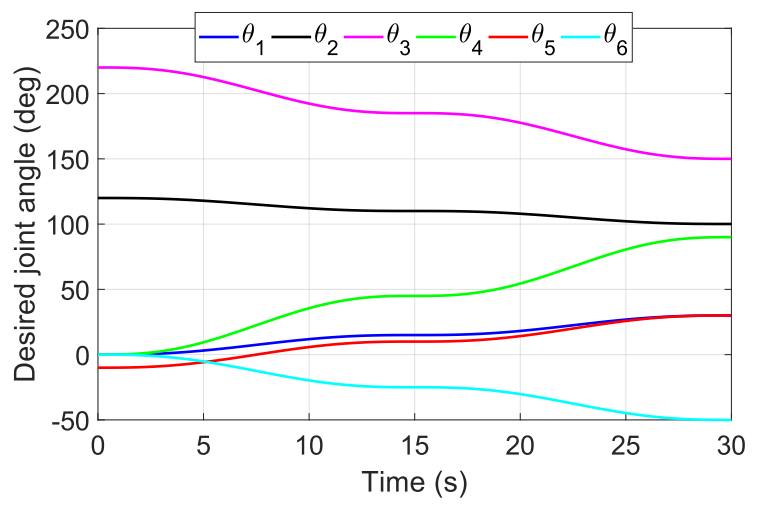

Fig. 5: Desired joint angles

TABLE II: D-H Parameters of the Satellite-manipulator System

\begin{tabular}{lllll}
\hline Link no. & $\alpha_{i-1}(\mathrm{deg})$ & $a_{i-1}(m)$ & $d_{i}(m)$ & $\theta_{i}(\mathrm{deg})$ \\
\hline 1 & 0.0 & 0.5 & 1.05 & 0.0 \\
2 & 90 & 0.0 & 0.0 & 120 \\
3 & 180 & 2.7 & 0.0 & 220 \\
4 & 90 & 0.0 & 2.16 & 0.0 \\
5 & -90 & 0.0 & 0.0 & -10 \\
6 & 90 & 0.0 & 0.35 & 0.0 \\
EE & 0.0 & 0.0 & 0.25 & 0.0 \\
\hline
\end{tabular}

TABLE III: Inertial Parameters of the Satellite-manipulator System

\begin{tabular}{llllllll}
\hline Parameter & Base & Link1 & Link2 & Link3 & Link4 & Link5 & Link6 \\
\hline$m(\mathrm{~kg})$ & 750 & 20 & 35 & 20 & 10 & 20 & 5.0 \\
${ }^{i} I_{x x}\left(\mathrm{~kg} \cdot \mathrm{m}^{2}\right)$ & 200 & 1.8 & 2.7 & 1.3 & 1.0 & 1.2 & 0.8 \\
${ }^{i} I_{x y}\left(\mathrm{~kg} \cdot \mathrm{m}^{2}\right)$ & 0.0 & 0.0 & 0.0 & 0.0 & 0.0 & 0.0 & 0.0 \\
${ }^{i} I_{x z}\left(\mathrm{~kg} \cdot \mathrm{m}^{2}\right)$ & 0.0 & 0.0 & 0.0 & 0.0 & 0.0 & 0.0 & 0.0 \\
${ }^{i} I_{y y}\left(\mathrm{~kg} \cdot \mathrm{m}^{2}\right)$ & 200 & 0.8 & 1.2 & 0.7 & 0.8 & 1.0 & 0.4 \\
${ }^{i} I_{y z}\left(\mathrm{~kg} \cdot \mathrm{m}^{2}\right)$ & 0.0 & 0.0 & 0.0 & 0.0 & 0.0 & 0.0 & 0.0 \\
${ }^{i} I_{z z}\left(\mathrm{~kg} \cdot \mathrm{m}^{2}\right)$ & 200 & 1.5 & 2.3 & 1.3 & 1.0 & 1.2 & 0.6 \\
${ }^{i} a_{x}(\mathrm{~m})$ & - & 0.0 & 1.35 & 0.0 & 0.0 & 0.0 & 0.0 \\
${ }^{i} a_{y}(\mathrm{~m})$ & - & 0.0 & 0.0 & -0.108 & 0.0 & -0.175 & 0.0 \\
${ }^{i} a_{z}(\mathrm{~m})$ & - & 0.0 & 0.0 & 0.0 & 0.0 & 0.0 & 0.125 \\
${ }^{i} b_{x}(\mathrm{~m})$ & 0.5 & 0.0 & 1.35 & 0.0 & 0.0 & 0.0 & 0.0 \\
${ }^{i} b_{y}(\mathrm{~m})$ & 0.0 & 0.0 & 0.0 & -0.108 & 0.0 & -0.175 & 0.0 \\
${ }^{i} b_{z}(\mathrm{~m})$ & 1.05 & 0.0 & 0.0 & 0.0 & 0.0 & 0.0 & 0.125 \\
\hline
\end{tabular}

TABLE IV: Controllers and Parameters

\begin{tabular}{ll}
\hline Controller & Control parameters \\
\hline CTC & $\boldsymbol{K}_{P}=\operatorname{diag}(0,0,0,7500,5000,5000,5000,5000,5000,50,50,8) \times 10^{3}$ \\
& $\boldsymbol{K}_{D}=\operatorname{diag}(0,0,0,500,500,500,500,500,500,20,80,1) \times 10^{2}$ \\
\hline \multirow{3}{*}{ CTC-SMC } & $\boldsymbol{\lambda}=\operatorname{diag}(0,0,0,1,1,1,1,1,1,1,1,1)$ \\
& $\boldsymbol{\boldsymbol { \Delta }}=[0,0,0,4,4,4,4,4,4,4,4,4]^{T} \times 10^{-3}$ \\
\hline \multirow{3}{*}{ TDE-SMC } & $\overline{\boldsymbol{H}}=\operatorname{diag}(0,0,0,50,50,50,5,5,5,0.5,0.5,0.5)$ \\
& $\boldsymbol{\lambda}=\operatorname{diag}(0,0,0,1,1,1,1,1,1,1,1,1)$ \\
& $\boldsymbol{g}=\operatorname{diag}(0,0,0,1,1,1,1,1,1,0.1,0.1,0.1) \times 10^{-1}$ \\
& $\overline{\boldsymbol{H}}=\operatorname{diag}(0,0,0,50,50,50,5,5,5,0.5,0.5,0.5)$ \\
& $\boldsymbol{K}_{P}=\operatorname{diag}(0,0,0,10,10,10,8,8,8,2,2,2)$ \\
& $\boldsymbol{K}_{D}=\operatorname{diag}(0,0,0,2,2,2,2,2,2,0.5,0.5,0.5)$ \\
& $\boldsymbol{K}_{1}=\operatorname{diag}(0,0,0,2,2,2,2,2,2,2,2,2)$ \\
\hline \multirow{3}{*}{ TDE-STC } & $\overline{\boldsymbol{H}}=\operatorname{diag}(0,0,0,50,50,50,5,5,5,0.5,0.5,0.5)$ \\
& $\boldsymbol{\lambda}=\operatorname{diag}(0,0,0,1,1,1,1,1,1,1,1,1)$ \\
& $\boldsymbol{K}_{1}=\operatorname{diag}(0,0,0,0.2,0.2,0.2,2,2,2,4,2,2) \times 10^{-1}$ \\
& $\boldsymbol{K}_{2}=\operatorname{diag}(0,0,0,0.08,0.08,0.08,3,3,3,4,2,2) \times 10^{-3}$ \\
\hline &
\end{tabular}

\section{A. Case 1: Model with friction}

The nominal model with joint friction is studied in this case. The manipulator friction parameters referred to 

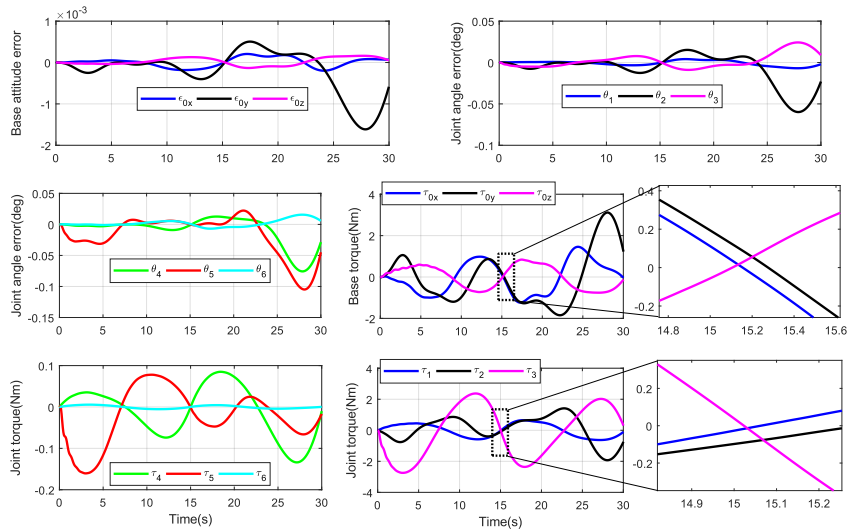

(a) Control performance of CTC
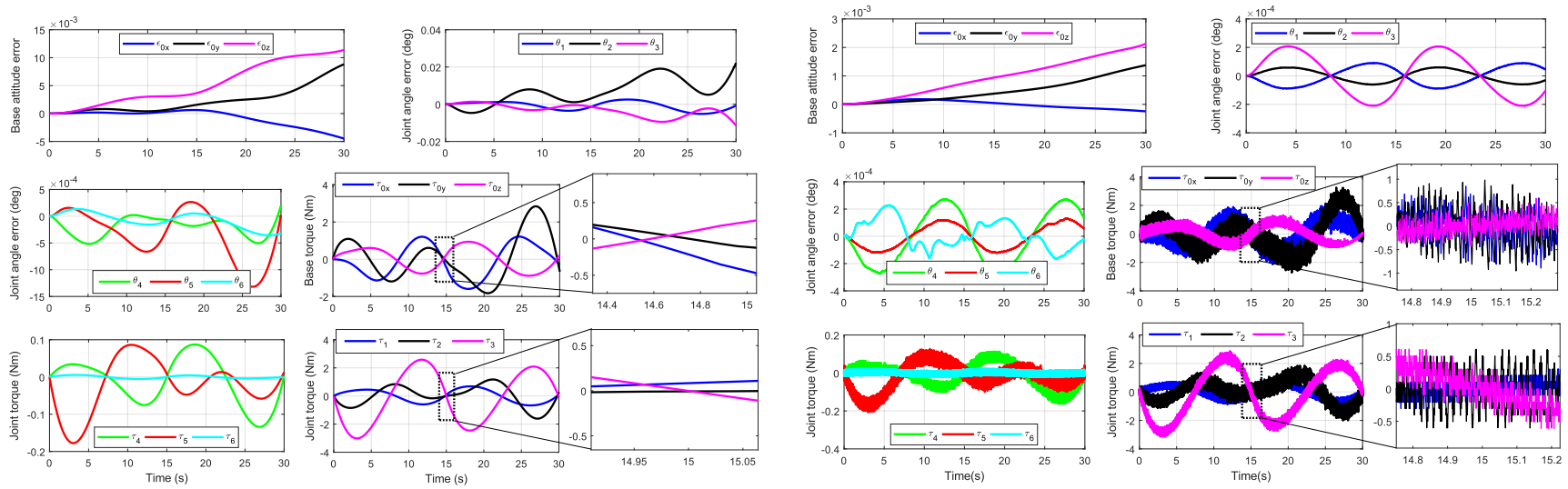

(b) Control performance of CTC-SMC

(c) Control performance of TDE-SMC
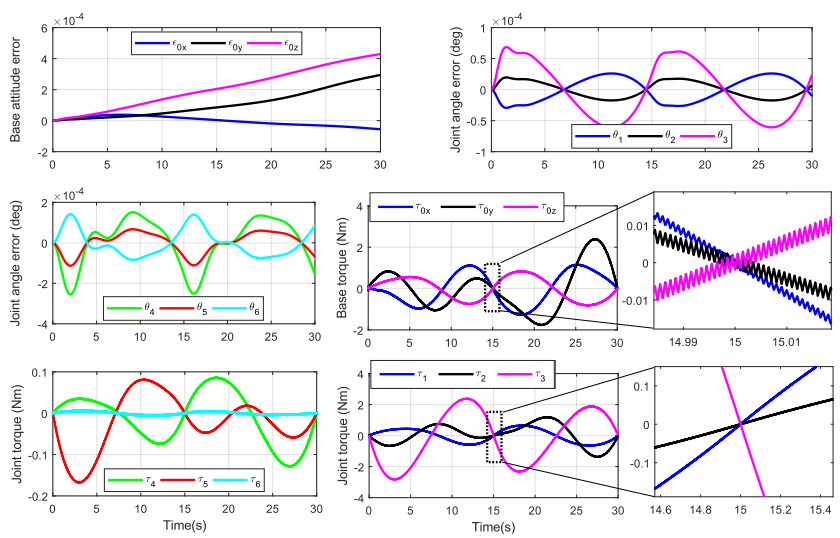

(d) Control performance of TDE-TSMC
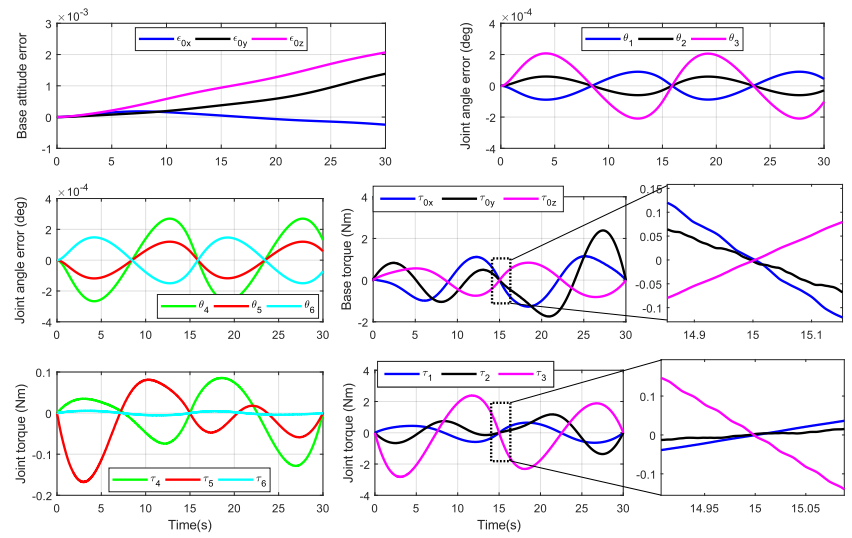

(e) Control performance of TDE-STC

Fig. 6: Control results of Case 1

[48] are set as: the Coulomb friction parameter $f_{C}=$ $\operatorname{diag}(2,2,2,2,2,2) \times 10^{-2}(\mathrm{Nm})$ and the viscous friction parameter $f_{V}=\operatorname{diag}(2,2,2,2,2,2) \times 10^{-1}(\mathrm{Nm} \cdot \mathrm{s} / \mathrm{rad})$. The corresponding control results of Case 1 are shown in Fig. 6.

\section{B. Case 2: Model with friction and disturbances}

On the basis of Case 1, external disturbances are considered in Case 2. As the environmental disturbances (for example gravity-gradient torque and magnetic torque) are cyclic in essence, they can be represented by sinusoids. Here, disturbance torque signals are assumed as

$$
\left\{\begin{array}{l}
f_{d 4}=f_{d 5}=f_{d 6}=0.01(\sin (\pi t)+\sin (1.5 \pi t)) \\
f_{d 7}=f_{d 8}=f_{d 9}=0.005(\sin (0.5 \pi t)+\sin (\pi t)) \\
f_{d 10}=f_{d 11}=f_{d 12}=0.002 \sin (0.5 \pi t)
\end{array}\right.
$$

where $f_{d i}(i=4,5,6)$, is the base disturbance, and $f_{d i}(i=$ $7, \cdots, 12)$, is the joint disturbance. The corresponding control results of Case 2 are shown in Fig. 7. 

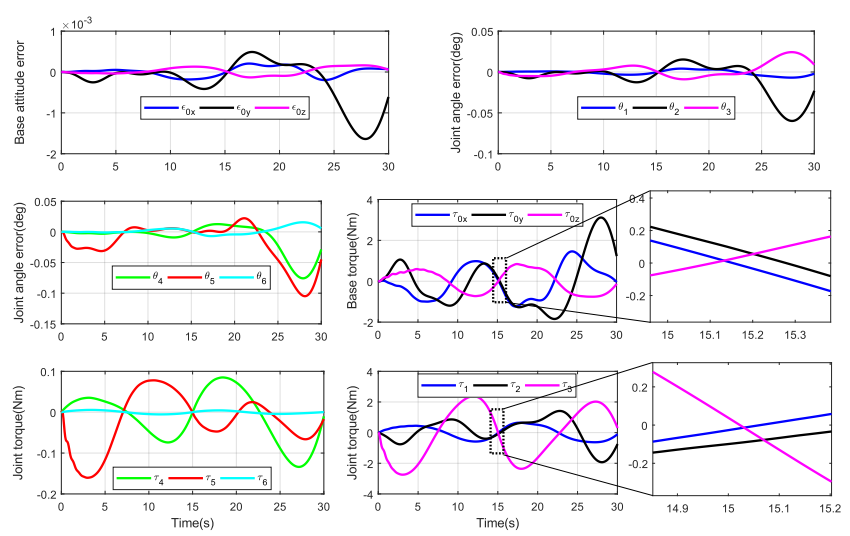

(a) Control performance of CTC
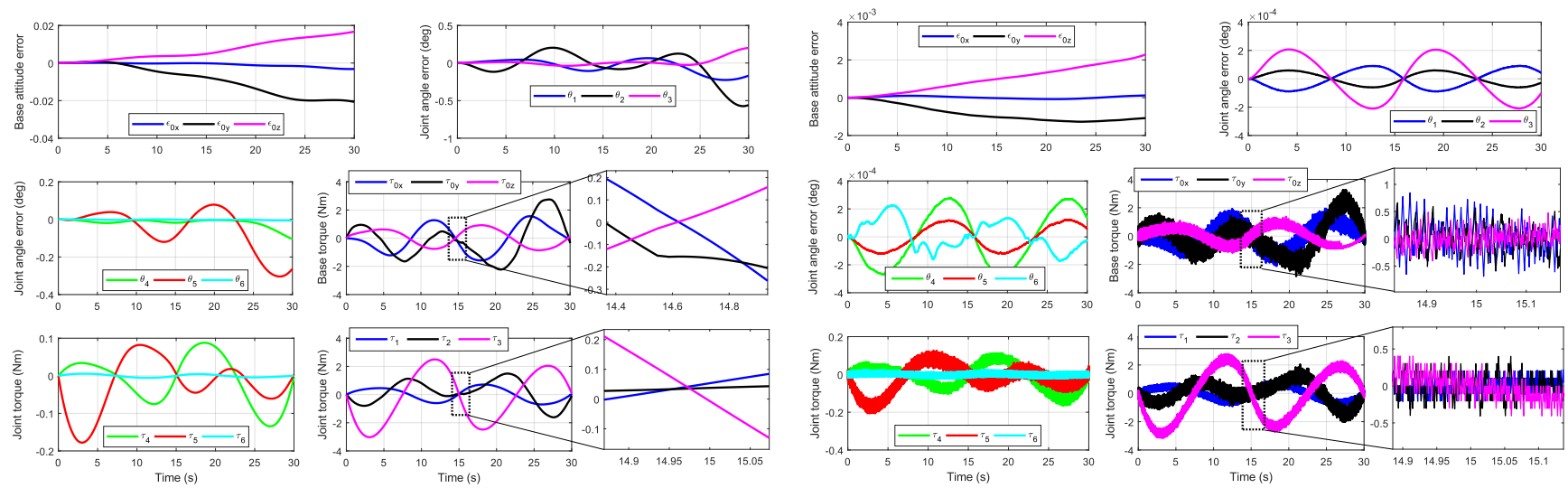

(b) Control performance of CTC-SMC

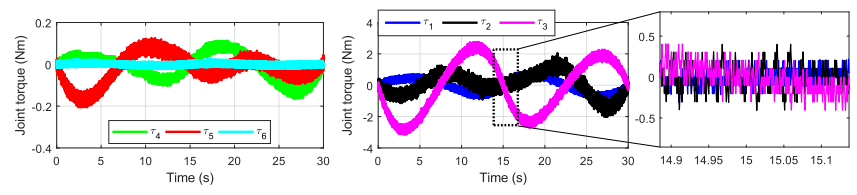

(c) Control performance of TDE-SMC
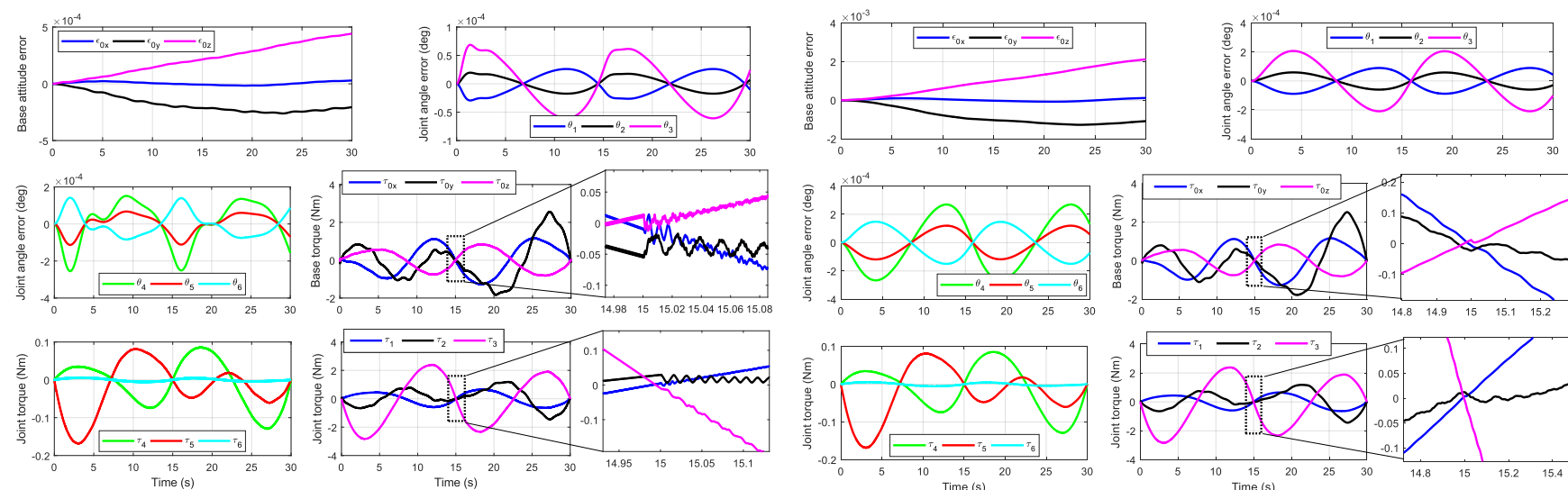

(d) Control performance of TDE-TSMC
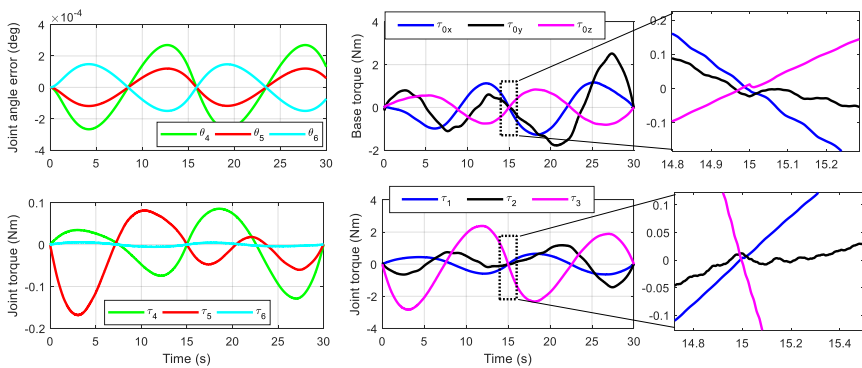

(e) Control performance of TDE-STC

Fig. 7: Control results of Case 2

C. Case 3: Model with friction, disturbances, and uncertainties

On the basis of Case 2, uncertainties are considered in Case 3 . We consider that the manipulator carries a vision payload to execute the monitoring mission. The system uncertainty is caused by the unknown payload, which leads to parameter changes of the end link. Herein, we assume that inertial parameters of the end link with the payload are changed to be: $m_{6}=10 \mathrm{~kg},{ }^{6} I_{6 x x}=1.2 \mathrm{~kg} \cdot \mathrm{m}^{2},{ }^{6} I_{6 y y}=0.6 \mathrm{~kg} \cdot \mathrm{m}^{2}$, and ${ }^{6} I_{6 z z}=1.0 \mathrm{~kg} \cdot \mathrm{m}^{2}$. Also, note that parameter changes are unknown to all the designed controllers. Meanwhile, planned trajectories still follow Fig. 5 and disturbances signals are still assumed as (61). The corresponding control results of Case 3 are shown in Fig. 8.

\section{Case 4: Model with different disturbances and uncertain-} ties

In this case, we consider that the space robot carries various unknown payloads and endures different disturbances. Two more situations are added to verify the robustness of the 

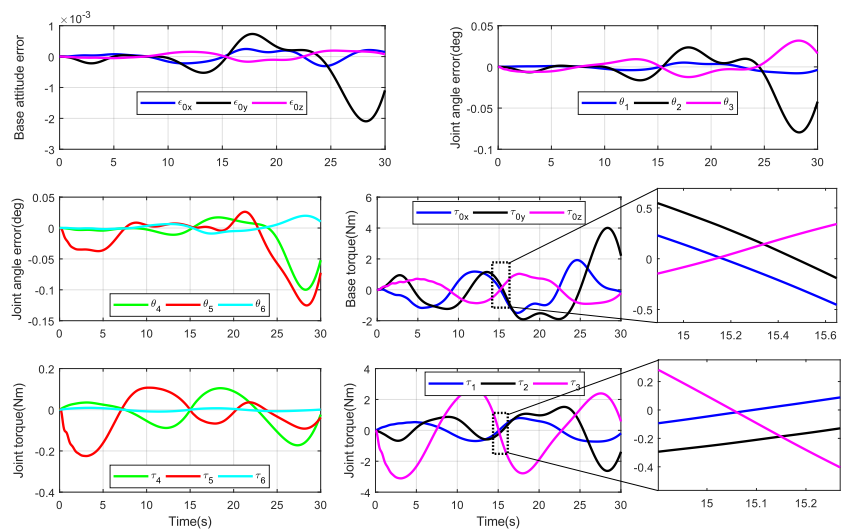

(a) Control performance of CTC
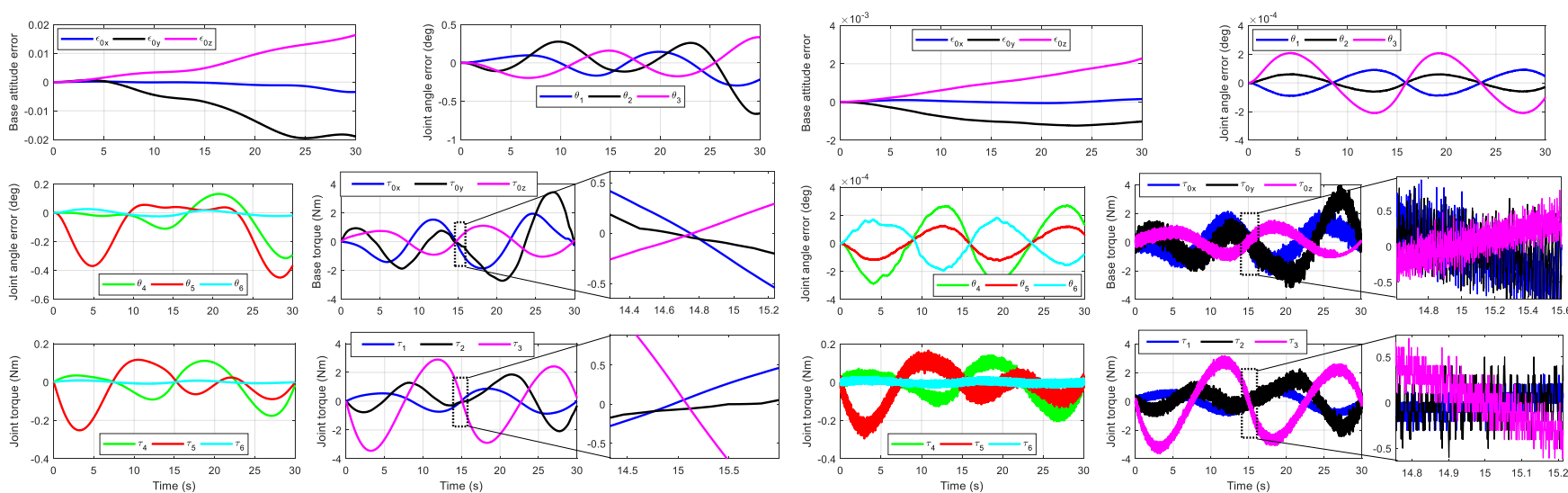

(b) Control performance of CTC-SMC

(c) Control performance of TDE-SMC
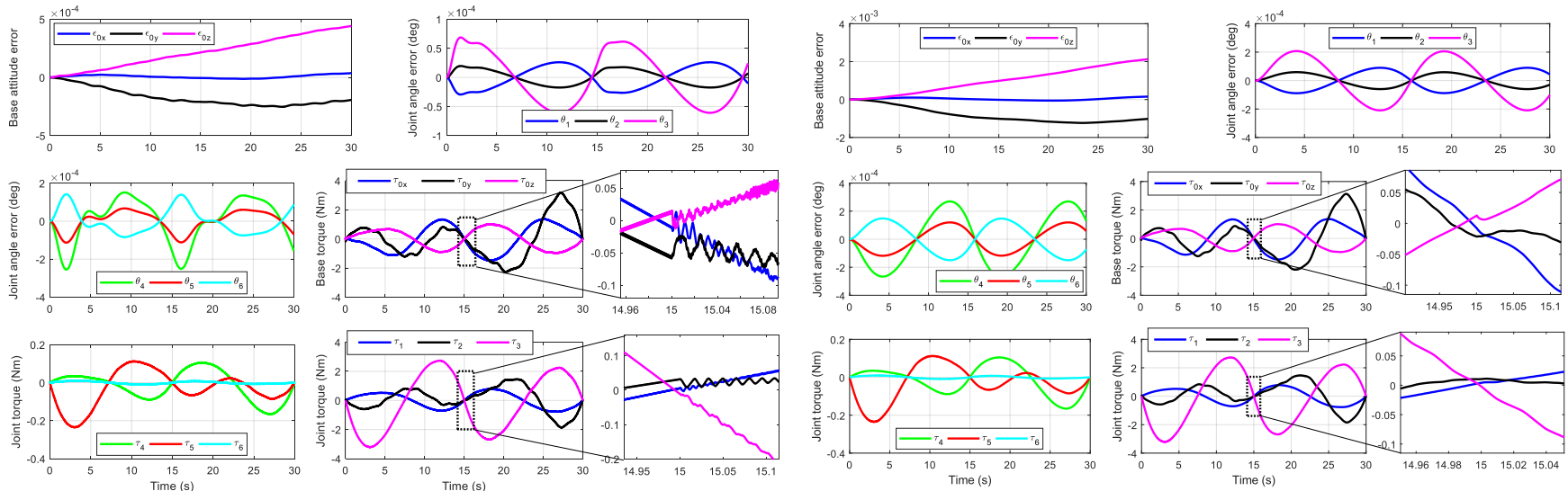

(d) Control performance of TDE-TSMC
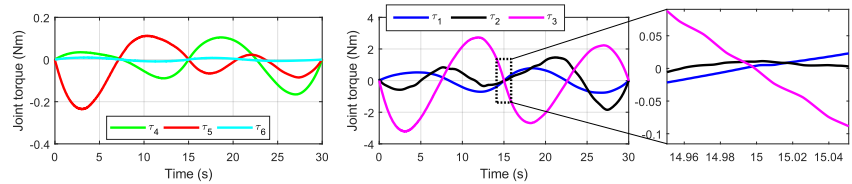

(e) Control performance of TDE-STC

Fig. 8: Control results of Case 3

proposed TDE-STC scheme. For the situation 1, disturbance signals are assumed as

$$
\left\{\begin{array}{l}
f_{d 4}=f_{d 5}=f_{d 6}=0.02(\sin (\pi t)+\sin (1.5 \pi t)) \\
f_{d 7}=f_{d 8}=f_{d 9}=0.01(\sin (0.5 \pi t)+\sin (\pi t)) \\
f_{d 10}=f_{d 11}=f_{d 12}=0.004 \sin (0.5 \pi t)
\end{array}\right.
$$

and inertial parameters of the end link with the payload are changed to be: $m_{6}=15 \mathrm{~kg},{ }^{6} I_{6 x x}=1.5 \mathrm{~kg} \cdot \mathrm{m}^{2},{ }^{6} I_{6 y y}=0.8 \mathrm{~kg}$. $\mathrm{m}^{2}$, and ${ }^{6} I_{6 z z}=1.4 \mathrm{~kg} \cdot \mathrm{m}^{2}$.
For the situation 2, disturbance signals are assumed as

$$
\left\{\begin{array}{l}
f_{d 4}=f_{d 5}=f_{d 6}=0.03(\sin (\pi t)+\sin (1.5 \pi t)) \\
f_{d 7}=f_{d 8}=f_{d 9}=0.015(\sin (0.5 \pi t)+\sin (\pi t)) \\
f_{d 10}=f_{d 11}=f_{d 12}=0.006 \sin (0.5 \pi t)
\end{array}\right.
$$

and inertial parameters of the end link with the payload are changed to be: $m_{6}=20 \mathrm{~kg},{ }^{6} I_{6 x x}=1.8 \mathrm{~kg} \cdot \mathrm{m}^{2},{ }^{6} I_{6 y y}=1.0 \mathrm{~kg}$. $\mathrm{m}^{2}$, and ${ }^{6} I_{6 z z}=1.6 \mathrm{~kg} \cdot \mathrm{m}^{2}$. The corresponding control results of Case 4 are shown in Fig. 9. 

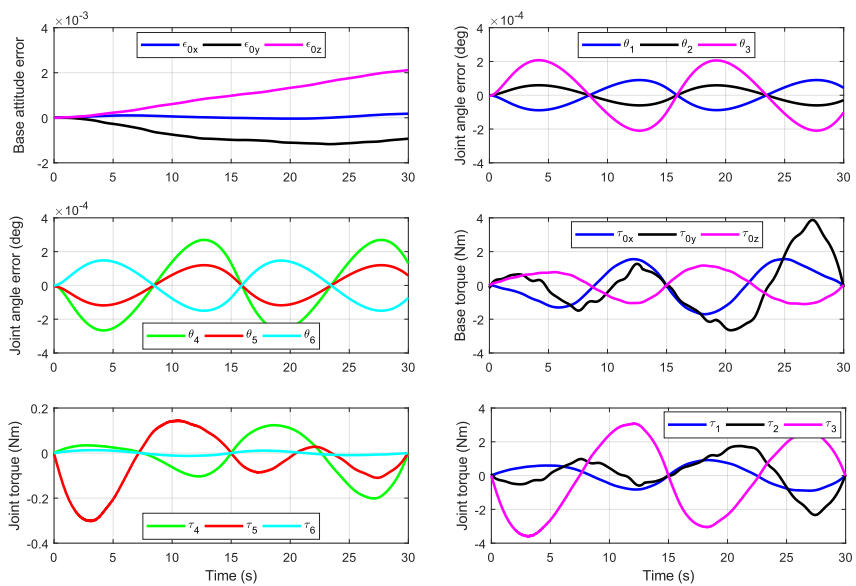

(a) TDE-STC in the situation 1
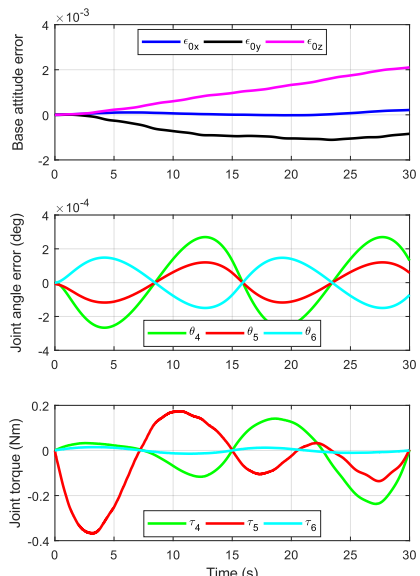

(b) TDE-STC in the situation 2
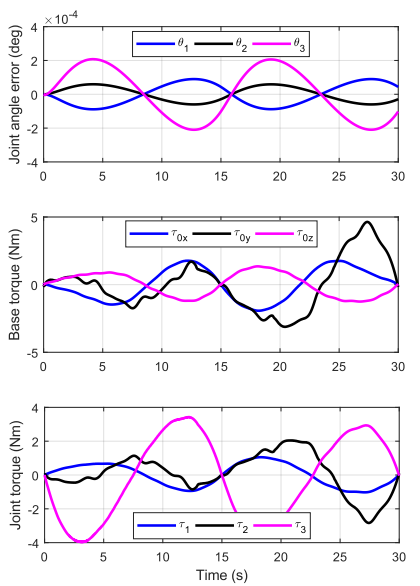

Fig. 9: Control results of Case 4

\section{E. Comparison and Discussion}

For the four case studies above, we make detailed statistics on control performances of each controller. The following quantitative indicators are adopted:

$$
\left\{\begin{array}{l}
\left\|e \epsilon_{0}\right\|_{R M S}=\frac{1}{3} \sqrt{\frac{1}{N_{S}} \sum_{i=1}^{N_{S}}\left\{e \epsilon_{0 x}^{2}(i)+e \epsilon_{0 y}^{2}(i)+e \epsilon_{0 z}^{2}(i)\right\}} \\
\left\|e \boldsymbol{\theta}_{j-(j+2)}\right\|_{R M S}=\frac{1}{3} \sqrt{\frac{1}{N_{S}} \sum_{i=1}^{N_{S}}\left\{e \theta_{j}^{2}(i)+e \theta_{j+1}^{2}(i)+e \theta_{j+2}^{2}(i)\right\}} \\
\left\|\tau_{0}\right\|_{R M S}=\frac{1}{3} \sqrt{\frac{1}{N_{S}} \sum_{i=1}^{N_{S}}\left\{\tau_{0 x}^{2}(i)+\tau_{0 y}^{2}(i)+\tau_{0 z}^{2}(i)\right\}} \\
\left\|\tau_{j-(j+2)}\right\|_{R M S}=\frac{1}{3} \sqrt{\frac{1}{N_{S}} \sum_{i=1}^{N_{S}}\left\{\tau_{j}^{2}(i)+\tau_{j+1}^{2}(i)+\tau_{j+2}^{2}(i)\right\}}
\end{array}\right.
$$

where $\left\|e \epsilon_{0}\right\|_{R M S}$ is the root mean square of the base attitude error; $\left\|e \boldsymbol{\theta}_{1-3}\right\|_{R M S}$ and $\left\|e \boldsymbol{\theta}_{4-6}\right\|_{R M S}$ are the root mean square of the first three and last three joint angle errors, respectively; $\left\|\tau_{0}\right\|_{R M S}$ is the root mean square of the base control torque; $\left\|\tau_{1-3}\right\|_{R M S}$ and $\left\|\tau_{4-6}\right\|_{R M S}$ are the root mean square of the first three and last three joint control torque, respectively; $i$ represents the $i$ th sampling time; and $N_{S}$ is the total number of sampling times.

Statistical results of case studies are listed in Table $\mathrm{V}$ and control performances of five different controllers are shown in Figs. 6-9. For CTC, its control output is continuous and its control performance is relatively stable, but the joint accuracy of the manipulator is not high. For CTC-SMC, due to the use of the boundary layer function, its control output is continuous enough but it has the lowest control accuracy (about two orders of magnitude lower than TDE-based controllers) and a poor anti-disturbance performance. For TDE-SMC, it can achieve a high control accuracy and a good anti-disturbance performance, but the discontinuous control output caused by the sign function is an obvious drawback. For TDE-TSMC, it achieves the highest control accuracy (especially in the last three joints) and a good anti-disturbance performance, but sometimes switching actions in small range (see the partial zoom in detail) occur in its control output in the process junction of the task. The main reason may be the use of the integral sliding surface, which is easy to accumulate errors and leads to oscillations in control. For TDE-STC, it achieves a relatively satisfied decoupling performance as good as TDE-SMC and its control output is absolutely continuous, smooth, and chatter-free. Moreover, results of Case 4 show the proposed controller can maintain its robustness when different disturbances and uncertainties occur in the model.

\section{Conclusion}

The attitude coupling issue of semi-floating space robots is investigated in this paper. The dynamics model of the space robot using the unit quaternion representation is derived by the composite rigid dynamics modelling approach. The TDE-STC scheme is designed to realize the attitude decoupling control efficiently and robustly. For verifying the effectiveness of the TDE-STC scheme, four simulation studies on a 3-D space robot system are conducted to compare the proposed controller with four existing controllers. Results demonstrate that the proposed controller can output continuous, smooth, and chatterfree control. Even, in the presence of different disturbances and uncertainties, the proposed controller can maintain its decoupling performance robustly without degrading its control accuracy.

\section{APPENDix A}

Proof of THE boundedness of $\dot{\varepsilon}$

The boundedness of $\dot{\varepsilon}$ is verified as follows. The TDE error is formulated as $\boldsymbol{\xi}(t)=\boldsymbol{N}(t)-\boldsymbol{N}(t-\delta)$. According to (33), (35), and (41), we have

$$
\left\{\begin{array}{c}
\boldsymbol{N}(t)-\boldsymbol{N}(t-\delta)=\overline{\boldsymbol{H}} \varepsilon(t) \\
\varepsilon(t)=\boldsymbol{u}_{S T C}(t)-\ddot{\boldsymbol{\rho}}(t)
\end{array}\right.
$$

Furthermore, combining (65), (27), (34), and (41), we can deduce

$$
\begin{aligned}
\boldsymbol{H}_{S}(t) \varepsilon(t)= & \left(\boldsymbol{H}_{S}(t)-\overline{\boldsymbol{H}}\right) \boldsymbol{u}_{S T C}(t) \\
& -\left(\boldsymbol{H}_{S}(t-\delta)-\overline{\boldsymbol{H}}\right) \ddot{\boldsymbol{\rho}}(t-\delta)+\boldsymbol{L} \\
\boldsymbol{L}=\boldsymbol{c}_{S}(t)- & \boldsymbol{c}_{S}(t-\delta)+\boldsymbol{f}_{d}(t)-\boldsymbol{f}_{d}(t-\delta) \\
+ & \boldsymbol{f}_{u}(t)-\boldsymbol{f}_{u}(t-\delta)+\boldsymbol{f}_{f}(t)-\boldsymbol{f}_{f}(t-\delta)
\end{aligned}
$$


TABLE V: Statistical Results of Case Studies

\begin{tabular}{llllllll}
\hline Controller & Case & $\left\|e \epsilon_{0}\right\|_{R M S}$ & $\left\|e \boldsymbol{\theta}_{1-3}\right\|_{R M S}$ & $\left\|e \boldsymbol{\theta}_{4-6}\right\|_{R M S}$ & $\left\|\boldsymbol{\tau}_{0}\right\|_{R M S}$ & $\left\|\boldsymbol{\tau}_{1-3}\right\|_{R M S}$ & $\left\|\boldsymbol{\tau}_{4-6}\right\|_{R M S}$ \\
\hline \multirow{3}{*}{ CTC } & 1 & $2.5642 \times 10^{-4}$ & 0.0107 & 0.0227 & 0.8772 & 0.9896 & 0.0454 \\
& 2 & $2.5922 \times 10^{-4}$ & 0.0107 & 0.0227 & 0.8773 & 0.9897 & 0.0454 \\
& 3 & $3.2715 \times 10^{-4}$ & 0.0137 & 0.0281 & 1.0617 & 1.1713 & 0.0606 \\
\hline \multirow{3}{*}{ CTC-SMC } & 1 & 0.0037 & 0.0055 & $3.3158 \times 10^{-4}$ & 0.9062 & 1.0030 & 0.0470 \\
& 2 & 0.0073 & 0.1139 & 0.0511 & 0.9622 & 1.0373 & 0.0470 \\
& 3 & 0.0070 & 0.1697 & 0.1143 & 1.1600 & 1.2310 & 0.0639 \\
\hline \multirow{2}{*}{ TDE-SMC } & 1 & $6.2914 \times 10^{-4}$ & $8.5157 \times 10^{-5}$ & $1.2593 \times 10^{-4}$ & 0.8328 & 0.9461 & 0.0489 \\
& 2 & $7.3128 \times 10^{-4}$ & $8.4997 \times 10^{-5}$ & $1.2709 \times 10^{-4}$ & 0.8318 & 0.9468 & 0.0490 \\
& 3 & $7.2389 \times 10^{-4}$ & $8.5018 \times 10^{-5}$ & $1.3153 \times 10^{-4}$ & 0.9924 & 1.1092 & 0.0628 \\
\hline \multirow{2}{*}{ TDE-TSMC } & 1 & $1.3647 \times 10^{-4}$ & $2.5458 \times 10^{-5}$ & $7.7319 \times 10^{-5}$ & 0.7991 & 0.9296 & 0.0451 \\
& 2 & $1.5397 \times 10^{-4}$ & $2.5458 \times 10^{-5}$ & $7.7319 \times 10^{-5}$ & 0.8003 & 0.9305 & 0.0451 \\
& 3 & $1.5253 \times 10^{-4}$ & $2.5458 \times 10^{-5}$ & $7.7314 \times 10^{-5}$ & 0.9635 & 1.0938 & 0.0600 \\
\hline \multirow{2}{*}{ TDE-STC } & 1 & $6.2950 \times 10^{-4}$ & $8.4955 \times 10^{-5}$ & $1.2742 \times 10^{-4}$ & 0.7988 & 0.9285 & 0.0450 \\
& 2 & $7.3142 \times 10^{-4}$ & $8.4956 \times 10^{-5}$ & $1.2742 \times 10^{-4}$ & 0.8027 & 0.9313 & 0.0451 \\
& 3 & $7.2380 \times 10^{-4}$ & $8.4959 \times 10^{-5}$ & $1.2742 \times 10^{-4}$ & 0.9665 & 1.0952 & 0.0601 \\
& $4-1$ & $7.0756 \times 10^{-4}$ & $8.4966 \times 10^{-5}$ & $1.2742 \times 10^{-4}$ & 1.1328 & 1.2610 & 0.0749 \\
\hline
\end{tabular}

Moreover,

$$
\varepsilon(t)=\boldsymbol{W}(t) \varepsilon(t-\delta)+\chi(t-\delta)
$$

where

$$
\begin{gathered}
\boldsymbol{W}(t)=\boldsymbol{E}-\boldsymbol{H}_{S}^{-1}(t) \overline{\boldsymbol{H}} \\
\chi(t-\delta)=\boldsymbol{H}_{S}^{-1}(t)\left\{\left(\boldsymbol{H}_{S}(t)-\boldsymbol{H}_{S}(t-\delta)\right) \ddot{\boldsymbol{\rho}}(t-\delta)+\boldsymbol{L}\right\} \\
+\boldsymbol{W}(t)(\boldsymbol{u}(t)-\boldsymbol{u}(t-\delta))
\end{gathered}
$$

Meanwhile, we know $\|\boldsymbol{W}(t)\|<1$ and $\varepsilon(t-\delta)$ is bounded, where the proof of the boundedness of $\varepsilon(t)$ can be referred to [45]. Thus, $\chi(t-\delta)$ is bounded, and $\dot{\varepsilon}(t)$ can be deduced by

$$
\begin{aligned}
\dot{\varepsilon}(t) & =\frac{1}{\delta}(\varepsilon(t)-\varepsilon(t-\delta)) \\
& =\frac{1}{\delta}(\boldsymbol{W}(t)-\boldsymbol{E}) \varepsilon(t-\delta)+\frac{1}{\delta} \chi(t-\delta) \\
& \leq \frac{1}{\delta}(\|\boldsymbol{W}(t)\|\|\varepsilon(t-\delta)\|+\|\varepsilon(t-\delta)\|+\|\chi(t-\delta)\|)
\end{aligned}
$$

As $\|\boldsymbol{W}(t)\|<1$ and $\varepsilon(t-\delta)$ and $\chi(t-\delta)$ are bounded, $\dot{\varepsilon}(t)$ is bounded.

\section{Appendix B}

Analysis of the LMI (51) in the Complex Frequency Domain

According to the circle criterion [49], the LMI (51) is solvable if the Nyquist plot of the transfer function falls within the circle with radius $\beta_{i}$, namely,

$$
\begin{gathered}
\boldsymbol{G}(s)=\boldsymbol{C}(\boldsymbol{E} s-\boldsymbol{A})^{-1} \boldsymbol{B} \\
\max _{\omega}|G(j \omega)|<\frac{1}{\beta_{i}}
\end{gathered}
$$

where $s$ represents the complex frequency, namely, $s=j \omega$, and $G(s)$ represents the transfer function. Making $|G(j \omega)|$ maximum is equivalent to making $|G(j \omega)|^{2}$ maximum.

$$
G(s)=\frac{1}{2 s^{2}+K_{1 i} s+K_{2 i}}
$$

$$
|G(j \omega)|^{2}=\frac{1}{\left(K_{2 i}-2 \omega^{2}\right)^{2}+\left(K_{1 i} \omega\right)^{2}}
$$

Its derivative is

$$
\frac{d}{d \omega}|G(j \omega)|^{2}=-8 \frac{\omega\left(2 \omega^{2}-K_{2 i}+\frac{K_{1 i}^{2}}{4}\right)}{\left\{\left(K_{2 i}-2 \omega^{2}\right)^{2}+\left(K_{1 i} \omega\right)^{2}\right\}^{2}}
$$

Furthermore, we can deduce that if $\frac{K_{1 i}^{2}}{4}-K_{2 i}>0, \max _{\omega}|G(j \omega)|$ reaches when $\omega=0$, or if $\frac{K_{1 i}^{2}}{4}-K_{2 i}<0, \max _{\omega}|G(j \omega)|$ reaches when $\omega^{2}=\frac{K_{2 i}}{2}-\frac{K_{1 i}^{2}}{8}$. Therefore, $\max _{\omega}|G(j \omega)|$ has two solutions as

$$
\max _{\omega}|G(j \omega)|^{2}=\left\{\begin{array}{c}
\frac{1}{K_{2 i}^{2}}, \text { if } \frac{K_{1 i}^{2}}{4}-K_{2 i}>0 \\
\frac{16}{K_{1 i}^{4}+8 K_{2 i} K_{1 i}^{2}-2 K_{1 i}^{3}}, \text { if } \frac{K_{1 i}^{2}}{4}-K_{2 i}<0
\end{array}\right.
$$

where we select the first solution to meet (51) when $K_{2 i}>\beta_{i}$ and $K_{1 i}^{2}>4 K_{2 i}$. We can solve the feasible $M$ to meet the LMI (51). Thus, the stability of the proposed TDE-STC controller is proven.

\section{AcKNOWLEDGMENT}

This research is supported by the National Key R\&D Program of China (Grant No. 2018YFB1304600), the National Natural Science Foundation of China (Grant No. 51775541 and No. 52075530), the China Postdoctoral Science Foundation (Grant No. 2020M670814), the CAS Interdisciplinary Innovation Team (Grant No. JCTD-2018-11), the State Key Laboratory of Robotics Foundation (Grant No. Y91Z0303), the Liaoning Provincial Natural Science Foundation (Grant No. 2020-MS-033), and the AiBle project co-financed by the European Regional Development Fund.

\section{REFERENCES}

[1] T. Kashangaki, "On-orbit damage detection and health monitoring of large space trusses-status and critical issues," in 32nd Structures, Structural Dynamics, and Materials Conference, 1991, pp. 2947-2958.

[2] P. J. From, J. T. Gravdahl, K. Y. Pettersen et al., Vehicle-manipulator systems. Springer, 2016.

[3] S. A. A. Moosavian and E. Papadopoulos, "Free-flying robots in space: an overview of dynamics modeling, planning and control," Robotica, vol. 25, no. 5, pp. 537-547, 2007. 
[4] X. Zhang, J. Liu, J. Feng, Y. Liu, and Z. Ju, "Effective capture of nongraspable objects for space robots using geometric cage pairs," IEEE/ASME Transactions on Mechatronics, vol. 25, no. 1, pp. 95-107, 2020.

[5] V. Muralidharan and M. R. Emami, "Rendezvous and attitude synchronization of a space manipulator," The Journal of the Astronautical Sciences, vol. 66, no. 1, pp. 100-120, 2019.

[6] H. Wang, D. Guo, H. Xu, W. Chen, T. Liu, and K. K. Leang, "Eyein-hand tracking control of a free-floating space manipulator," IEEE Transactions on Aerospace and Electronic Systems, vol. 53, no. 4, pp. 1855-1865, 2017.

[7] Y. Zhang, W. Liang, H. He, and J. Tan, "Wearable heading estimation for motion tracking in health care by adaptive fusion of visual-inertial measurements," IEEE journal of biomedical and health informatics, vol. 22, no. 6, pp. 1732-1743, 2018.

[8] Q. Gao, J. Liu, and Z. Ju, "Robust real-time hand detection and localization for space human-robot interaction based on deep learning," Neurocomputing, vol. 390, pp. 198-206, 2020.

[9] X. Zhang and J. Liu, "Autonomous trajectory planner for space telerobots capturing space debris under the teleprogramming framework," Advances in Mechanical Engineering, vol. 9, no. 9, pp. 1-13, 2017.

[10] H. Sone and D. Nenchev, "Reactionless camera inspection with a free-flying space robot under reaction null-space motion control," Acta Astronautica, vol. 128, pp. 707-721, 2016.

[11] A. M. Giordano, C. Ott, and A. Albu-Schäffer, "Coordinated control of spacecraft's attitude and end-effector for space robots," IEEE Robotics and Automation Letters, vol. 4, no. 2, pp. 2108-2115, 2019.

[12] Y. Xu and H.-Y. Shum, "Dynamic control and coupling of a free-flying space robot system," Journal of Robotic systems, vol. 11, no. 7, pp. 573-589, 1994.

[13] Y. Zhou, J. Luo, and M. Wang, "Dynamic coupling analysis of multi-arm space robot," Acta Astronautica, vol. 160, pp. 583-593, 2019.

[14] W. Xu, J. Peng, B. Liang, and Z. Mu, "Hybrid modeling and analysis method for dynamic coupling of space robots," IEEE Transactions on Aerospace and Electronic Systems, vol. 52, no. 1, pp. 85-98, 2016.

[15] D. N. Nenchev, K. Yoshida, P. Vichitkulsawat, and M. Uchiyama, "Reaction null-space control of flexible structure mounted manipulator systems," IEEE Transactions on Robotics and Automation, vol. 15, no. 6 , pp. 1011-1023, 1999

[16] S. Xu, H. Wang, D. Zhang, and B. Yang, "Adaptive zero reaction motion control for free-floating space manipulators," IEEE Transactions on Aerospace and Electronic Systems, vol. 52, no. 3, pp. 1067-1076, 2016.

[17] L. Zong, M. R. Emami, and J. Luo, "Reactionless control of free-floating space manipulators," IEEE Transactions on Aerospace and Electronic Systems, vol. 56, no. 2, pp. 1490-1503, 2019.

[18] P. Huang, Y. Xu, and B. Liang, "Dynamic balance control of multi-arm free-floating space robots," International Journal of Advanced Robotic Systems, vol. 2, no. 2, p. 13, 2005.

[19] R. W. Longman, R. E. Lindbergt, and M. F. Zedd, "Satellite-mounted robot manipulators-new kinematics and reaction moment compensation," The International Journal of Robotics Research, vol. 6, no. 3, pp. 87-103, 1987.

[20] A. Antonello, A. Valverde, and P. Tsiotras, "Dynamics and control of spacecraft manipulators with thrusters and momentum exchange devices," Journal of Guidance, Control, and Dynamics, vol. 42, no. 1, pp. 15-29, 2019.

[21] H. S. Jayakody, L. Shi, J. Katupitiya, and N. Kinkaid, "Robust adaptive coordination controller for a spacecraft equipped with a robotic manipulator," Journal of Guidance, Control, and Dynamics, pp. 2699-2711, 2016.

[22] L. Shi, S. Kayastha, and J. Katupitiya, "Robust coordinated control of a dual-arm space robot," Acta Astronautica, vol. 138, pp. 475-489, 2017.

[23] L. Zong, M. Reza Emami, and V. Muralidharan, "Concurrent rendezvous control of underactuated space manipulators," Journal of Guidance, Control, and Dynamics, vol. 42, no. 11, pp. 2501-2510, 2019.

[24] L. Zong and M. R. Emami, "Concurrent base-arm control of space manipulators with optimal rendezvous trajectory," Aerospace Science and Technology, pp. 1-10, 2020.

[25] Y. Zhu, J. Qiao, and L. Guo, "Adaptive sliding mode disturbance observer-based composite control with prescribed performance of space manipulators for target capturing," IEEE Transactions on Industrial Electronics, vol. 66, no. 3, pp. 1973-1983, 2018.

[26] X. Zhang, J. Liu, Q. Gao, and Z. Ju, "Adaptive robust decoupling control of multi-arm space robots using time-delay estimation technique," Nonlinear Dynamics, vol. 100, no. 3, pp. 2449-2467, 2020.
[27] B. Xiao, Q. Hu, and Y. Zhang, "Adaptive sliding mode fault tolerant attitude tracking control for flexible spacecraft under actuator saturation," IEEE Transactions on Control Systems Technology, vol. 20, no. 6, pp. 1605-1612, 2011.

[28] Y. Shtessel, C. Edwards, L. Fridman, and A. Levant, Sliding mode control and observation. Springer, 2014.

[29] S. Yu, X. Yu, B. Shirinzadeh, and Z. Man, "Continuous finite-time control for robotic manipulators with terminal sliding mode," Automatica, vol. 41, no. 11, pp. 1957-1964, 2005.

[30] M. Jin, Y. Jin, P. H. Chang, and C. Choi, "High-accuracy tracking control of robot manipulators using time delay estimation and terminal sliding mode," International Journal of Advanced Robotic Systems, vol. 8, no. 4, p. 33, 2011.

[31] S. Ding, J. Wang, and W. X. Zheng, "Second-order sliding mode control for nonlinear uncertain systems bounded by positive functions," IEEE Transactions on Industrial Electronics, vol. 62, no. 9, pp. 5899-5909, 2015.

[32] A. Ferrara and M. Rubagotti, "A sub-optimal second order sliding mode controller for systems with saturating actuators," IEEE Transactions on Automatic Control, vol. 54, no. 5, pp. 1082-1087, 2009.

[33] A. Levant, "Principles of 2-sliding mode design," Automatica, vol. 43, no. 4 , pp. $576-586,2007$

[34] — "Sliding order and sliding accuracy in sliding mode control," International journal of control, vol. 58, no. 6, pp. 1247-1263, 1993.

[35] Y. Kali, M. Saad, K. Benjelloun, and C. Khairallah, "Super-twisting algorithm with time delay estimation for uncertain robot manipulators," Nonlinear Dynamics, vol. 93, no. 2, pp. 557-569, 2018.

[36] L. Derafa, A. Benallegue, and L. Fridman, "Super twisting control algorithm for the attitude tracking of a four rotors uav," Journal of the Franklin Institute, vol. 349, no. 2, pp. 685-699, 2012.

[37] F. Zhang and P. Huang, "Releasing dynamics and stability control of maneuverable tethered space net," IEEE/ASME Transactions on Mechatronics, vol. 22, no. 2, pp. 983-993, 2016.

[38] Y. Zhao, F. Zhang, P. Huang, and X. Liu, "Impulsive super-twisting sliding mode control for space debris capturing via tethered space net robot," IEEE Transactions on Industrial Electronics, vol. 67, no. 8, pp. 6874-6882, 2019.

[39] X. Zhang and J. Liu, "Effective motion planning strategy for space robot capturing targets under consideration of the berth position," Acta Astronautica, vol. 148, pp. 403-416, 2018.

[40] R. Featherstone, Rigid body dynamics algorithms. Springer, 2014.

[41] W. Huo, Robot dynamics and control. Higher Education Press, Beijing, 2005.

[42] M. Jin, J. Lee, and N. G. Tsagarakis, "Model-free robust adaptive control of humanoid robots with flexible joints," IEEE Transactions on Industrial Electronics, vol. 64, no. 2, pp. 1706-1715, 2016.

[43] S. Roy and I. N. Kar, "Adaptive sliding mode control of a class of nonlinear systems with artificial delay," Journal of the franklin Institute, vol. 354 , no. 18 , pp. 8156-8179, 2017.

[44] M. Jin, S. H. Kang, and P. H. Chang, "Robust compliant motion control of robot with nonlinear friction using time-delay estimation," IEEE Transactions on Industrial Electronics, vol. 55, no. 1, pp. 258-269, 2008.

[45] M. Jin, J. Lee, P. H. Chang, and C. Choi, "Practical nonsingular terminal sliding-mode control of robot manipulators for high-accuracy tracking control," IEEE Transactions on Industrial Electronics, vol. 56, no. 9, pp. 3593-3601, 2009

[46] J. A. Moreno, "A linear framework for the robust stability analysis of a generalized super-twisting algorithm," in 2009 6th International Conference on Electrical Engineering, Computing Science and Automatic Control (CCE). IEEE, 2009, pp. 1-6.

[47] A. Dávila, J. A. Moreno, and L. Fridman, "Optimal lyapunov function selection for reaching time estimation of super twisting algorithm," in Proceedings of the 48h IEEE Conference on Decision and Control $(C D C)$ held jointly with 2009 28th Chinese Control Conference. IEEE, 2009, pp. 8405-8410.

[48] H. Chaoui, P. Sicard, and W. Gueaieb, "Ann-based adaptive control of robotic manipulators with friction and joint elasticity," IEEE Transactions on Industrial Electronics, vol. 56, no. 8, pp. 3174-3187, 2009.

[49] H. K. Khalil and J. W. Grizzle, Nonlinear systems. Prentice Hall Upper Saddle River, NJ, 2002. 